\title{
Impact of Grid interconnected micro-hydro based mini-grid system on Local Distribution Grid: A Case study of Taplejung mini-grid, Nepal
}

\author{
Rajmani Bajagain, Nawraj Bhattarai, Tek Raj Subedi
}

\begin{abstract}
Electricity is essential for accelerating the economic development of any country and is also taken as an important input to improve quality of life. Micro hydro power plants are very much successful for rural electrification in Nepal as compared to other countries in the world. Till 2018, more than 3000 MHPs aggregating to $35 \mathrm{MW}$ of generation capacity have been developed in Nepal. It cost about NPR17-25 per $\mathrm{kWh}$ for Nepal Electricity Authority (NEA)'s grid to deliver electricity in the rural hilly areas (depending on the distance) whereas it cost about 9-15 per KWH via a 50-100 KW MHP that is connected to the grid. So, In Nepal, it is more economically feasible for interconnected the existing isolated mini grid Or MHPs to the Local Distribution grid. Mini-Grids are electricity distribution systems containing loads \& distributed energy resources within clearly defined electrical boundaries acting as a single controllable entity with respect to the utility grid that can be operated in a controlled and coordinated way to operate in both grid connected or island mode. This paper presents the impacts of installing mini-grid on a distribution grid. The work is focused on analyzing the impact of mini-grid installation on distribution grid operation including voltage analysis and power losses of the system. Different DG penetration levels, locations and the impacts of installing one large-scale DG on the main distribution line and distributing it several locations on voltage profile and losses are explored.

The research involves several case studies that explore the impacts of installing distributed generation (DG) on a distribution network operation including the voltage profile and losses of the system. Water Turbine Generators are introduced as Distributed Generators (DGs) at various nodes and the impacts that DG produces on power losses and voltage profile is studied. Simulated results obtained using load flow are presented and discussed.
\end{abstract}

Keywords - Voltage Profile, Losses, Local Distribution Network, Economic Development

\section{INTRODUCTION}

Electricity plays a key role in the modern society because of its versatility with respect to the input-energy form. Electric power in the form of synchronized alternating current is generated by generating plants, and delivered to users as per requirements. The electricity travels at about the speed of light and is consumed within a fraction of a second after it is generated [18]. Nowadays electricity generation from Small, Mini and Micro hydropower plants is becoming popular. In many remote hilly areas these power plants are generating power in isolated mode i.e., not grid connected, and supplying power in local areas due to unavailability of Distribution Grid[8]. In case of shut down of plant, power supply to those areas is affected. Hence to increase the reliability of the power supply to such areas, Mini Grid can be very effective solution [5].

The analysis by AEPC shows that the percentage of population having access of electricity till date is $87.55 \%$ indicating $12.45 \%$ population deprived of access to electricity. Rural electrification serves 9.75\% (Solar 6.25\% and Micro hydro $3.5 \%$ ) of the population while NEA grid serves $77.8 \%$ [1].

Nepal is known for its successful rural electrification efforts through community owned and managed standalone MHP that have helped transform a large part of its remote and hilly districts [12]. Nepal's green energy, which totals more than 36 MW today, has not only brought electricity to more than 350,000 families in remote areas away from the grid, it has created an environment conducive for new economic activities, relieved people of drudgeries, improved their health and helped better children's education[9].

The adoption of renewable energy has been increasing in a very encouraging way all over the world [7]. The lack of productive activities in the rural areas have also resulted in underutilization of the mini grid or MHPs. During underutilization, the loss of energy can be as high as $72 \%$. Connecting to the Local distribution grid help to divert this extra energy via the Local distribution grid \& reduce losses. Likewise, during under production, mini grid or MHPs can also receive power from the Local distribution grid.

Load shedding of Nepal is being managed by significant import of electricity from India, thus increasing the trade deficit of Nepal [11].

Electrification in rural areas by grid extension seems particularly unfeasible in the country because of high transmission/distribution cost, low consumption per household and less number of consumers/sparse load [6].If isolated mini grids will be connected to the Local distribution grid, they could feed surplus electricity to the Local distribution grid and receive deficit energy from the Local distribution grid [16].

The government policy for off grid/no connection regarding national electrification plan needs to be revised and rethought for future development and growth of this sector [9].The 
existing mini grid power will contribute to stabilize the weak national grid that will serve to the rural villages [16].

One of the greatest and the most obvious problem that Nepal and the other developing countries are facing today is the increasing demand of electricity and its poor supply [15]. The rising gap between demand and supply of electricity is the major factor of concern to developing countries like Nepal. At the same time, customers often suffer from poor power quality such as variations in voltage or electrical flow that results from a variety of factors, including poor switching operations in the network, voltage dips, interruptions, transients, and network disturbances from loads [14]. The DG can be placed at several locations depending upon the network to address these issues. Overall, DG proponents highlight the inefficiency of the existing large-scale electrical transmission and distribution network [10]. Properly coordinated DG can improve the voltage profile of the system and enhance the power system stability. Placing the DG at optimal location can reduce the losses on the feeder. With the growing use of DG, [12] it is critical to study its impacts on the distribution system operation.

The power system is prone to failures and disturbances due to weather related issues, accidents, human errors [14] [15]. Having the DG as a backup source ensures the reliability of power supply which is critical to business and industry. The overall reliability of the system can be improved. One of the main advantages of DG is their close proximity to the customer loads they are serving [12]. DG can play an important role in improving the reliability of the current grid, reducing the losses, providing voltage support and improving power quality [15]. The major obstacle for the distributed generation has been the high cost. However, the costs have decreased significantly over the past 20 years [3]. The distributed generation also reduces greenhouse gas emission addressing pollutant concerns by providing clean and efficient energy [17]. Distributed generation is the key to meeting growing demands of electricity and provides benefits to customers, utility and market.

Interconnecting a DG to the distribution feeder can have significant effects on the system such as power flow, voltage regulation, reliability etc. A DG installation changes traditional characteristics of the distribution system. Most of the distribution systems are designed such that the power flows in one direction [15]. The installation of a DG introduces another source in the system. When the DG power is more than the downstream load, it sends power upstream reversing the direction of power flow and at some point between the DG and substation; the real power flow is zero due to back flow of power from DG [14].

The DG installation can impact the overall voltage profile and losses of the system. Inclusion of DG can improve feeder voltage of distribution networks in areas where voltage dip or blackouts are of concern for utilities[10].

\section{METHODOLOGY}

It specifically employs the case study approach to explore the power generation and surplus power exported from mini-grid to the nearest distribution grid. The analytical software ETAP (Electrical Transient Analyzer Program) is used to study grid impact. Amarpur Substation is selected to analyses the grid impact upon integration of mini grid.

\section{A. $\quad$ Case Study Area}

The study area for this paper is chosen as Taplejung District of Nepal. Taplejung lies approximately $850 \mathrm{~km}$ North East of Kathmandu. Taplejung district lies in the Mechi zone of Provience no 1 of Nepal. The economy of the district is dependent on the agricultural production in which water, one of the known natural resources of the district, plays a great role. Taplejung district is one of the district which is recently powered by central grid electricity. There are 6 Micro Hydro Project in the proposed area currently operating in islanding mode. The house mark in the figure represents the isolated MHP. These micro hydropower projects are proposed for interconnection forming $11 \mathrm{kV}$ transmission line in connected to Local Distribution grid. The layout of MHP in isolation mode is presented in the figure below:

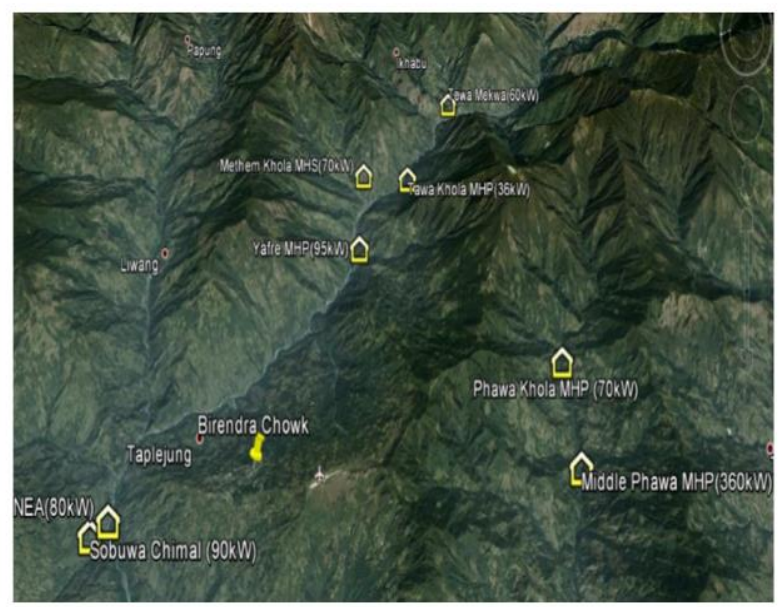

Fig.1:- Layout of MHP in isolation mode (district development commiunity, 2016)

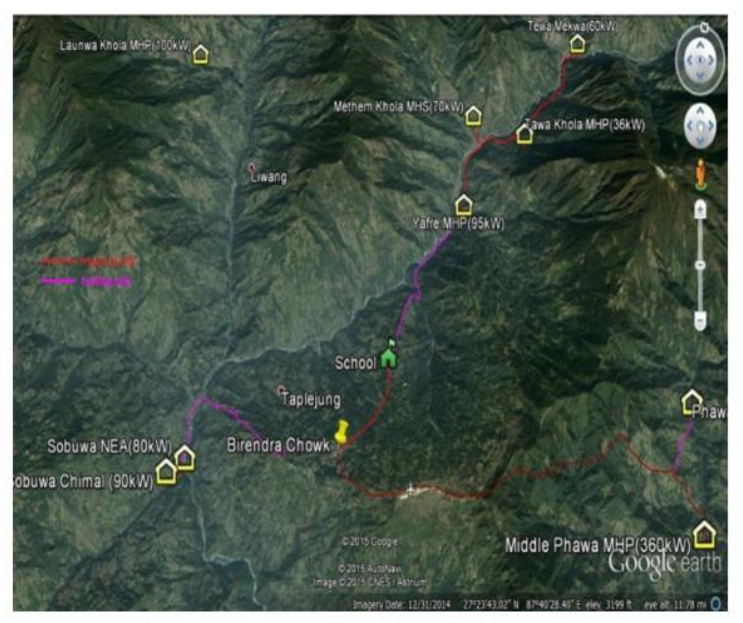

Figure2:- Interconnection View of Taplejung Mini Grid (district development commiunity, 2016) 
Taplejung district is one of the districts recently powered by central grid electricity. $33 \mathrm{KV}$ Line is connected from Amarpur132/33 KV, 30 MVA Power Transformer S/S of Pachathar districit about $40 \mathrm{~km}$ with Dog Conductor. 6/8 MVA Power Transformer is installed in Phuling Bazar. The load of Taplejung is 2 MVA (NEA, 2018/19).

\section{Data Collection}

Basic data regarding the mini grids are collected which includes length of grid, single line diagram, capacity of generator, type of conductor, transformer (Gyawali, 2014).

\section{Modelling}

The model developed containing Taplejung substation distribution network, NEA distribution and transmission systems with step-up transformer for grid impact study is shown in Figure 3. The simulation study is done with following assumptions (NEA, 2018/19).

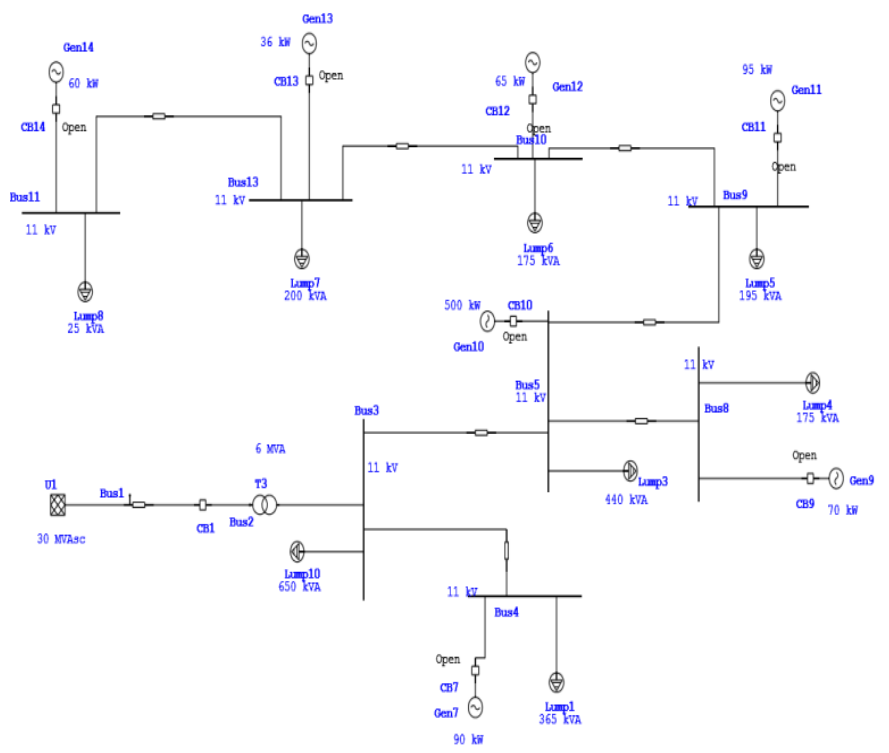

Fig.3 Single Line Diagram of System

- $\quad$ The power generation from each generators is $90 \%$.

- $\quad$ The conductor used for $11 \mathrm{kV}$ and $33 \mathrm{kV}$ are RABBIT and DOG conductor respectively.

- Amarpur substation $33 \mathrm{kV}$ bus is considered as slack bus. cases.

a)

In Case-1, Load flow analysis for Local Distribution Grid Only.

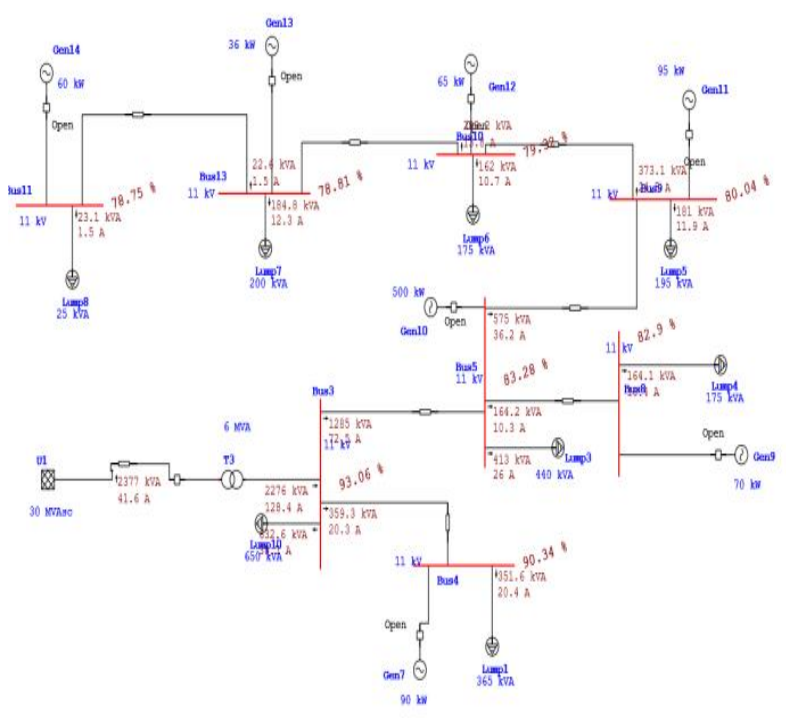

Fig.4 Load flow analysis of LDG System

Table 1: Simulation Data for Local Distribution Grid (LDG) supply only

\begin{tabular}{|c|c|c|c|c|c|c|c|}
\hline \multicolumn{8}{|c|}{ LOAD DATA } \\
\hline Rating/Limit & Rated kV & kW & kvar & Amp & $\% \mathrm{PF}$ & $\begin{array}{l}\% \\
\text { Loading }\end{array}$ & Vtermal \\
\hline $365 \mathrm{kVA}$ & 11 & 298.8 & 185.2 & 20.43 & 85 & 106.6 & 90.34 \\
\hline $440 \mathrm{kVA}$ & 11 & 351.1 & 217.6 & 26.03 & 85 & 112.7 & 83.28 \\
\hline $175 \mathrm{kVA}$ & 11 & 139.4 & 86.42 & 10.39 & 85 & 113.1 & 82.9 \\
\hline $195 \mathrm{kVA}$ & 11 & 153.8 & 95.34 & 11.87 & 85 & 116 & 80.04 \\
\hline $175 \mathrm{kVA}$ & 11 & 137.7 & 85.35 & 10.72 & 85 & 116.7 & 79.32 \\
\hline $200 \mathrm{kVA}$ & 11 & 157.1 & 97.37 & 12.31 & 85 & 117.3 & 78.81 \\
\hline $25 \mathrm{kVA}$ & 11 & 19.64 & 12.17 & 1.54 & 85 & 117.3 & 78.75 \\
\hline $650 \mathrm{kVA}$ & 11 & 537.7 & 333.2 & 35.68 & 85 & 104.6 & 93.06 \\
\hline & & & & & & & 666.5 \\
\hline & & & & & & & 83.3125 \\
\hline & & & & & $\begin{array}{l}\text { 3Phase Load } \\
\text { Terminal Voltage }\end{array}$ & & 333.2 \\
\hline \multicolumn{8}{|c|}{ SOURCE DATA } \\
\hline ID & $\begin{array}{c}\text { Terminal } \\
\text { Bus }\end{array}$ & Rating/Limit & Rated kV & MW & Mvar & Amp & $\% \mathrm{PF}$ \\
\hline U1 & Bus1 & $30 \mathrm{MVA}$ & 33 & 2.002 & 1.283 & 41.59 & 84.2 \\
\hline
\end{tabular}

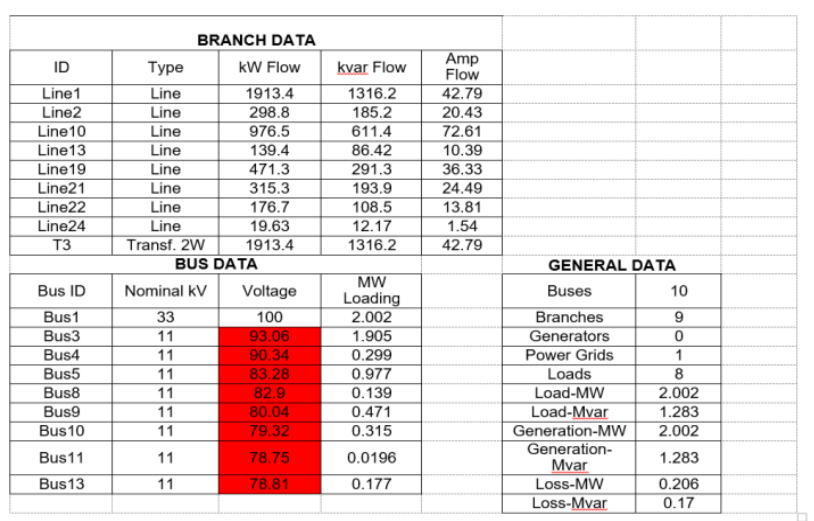

b) In Case-2, simulation is done with Local Distribution grid and Chimal plant (90 KW). 
International Journal of Engineering and Applied Sciences (IJEAS)

ISSN: 2394-3661, Volume-7, Issue-4, April 2020

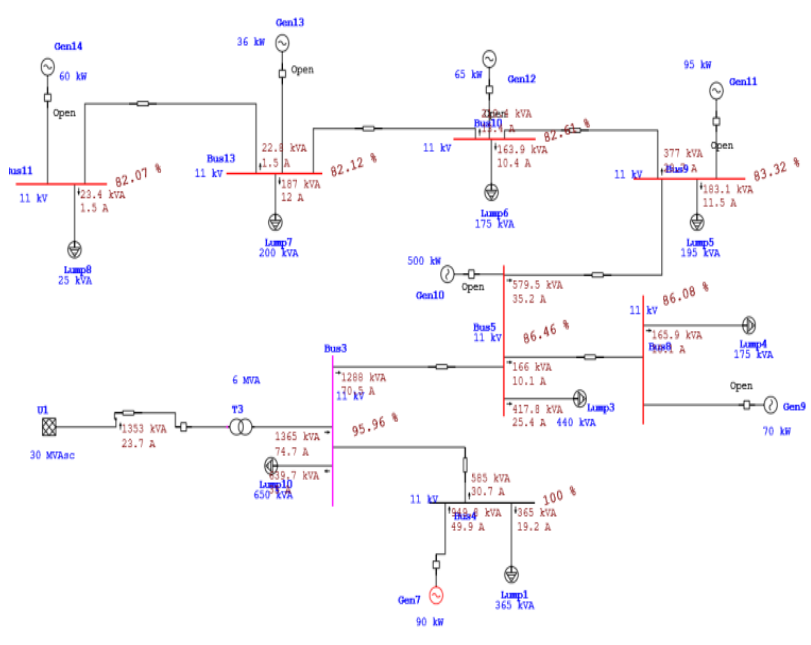

Figure 5: Load flow analysis for Local Distribution Grid (LDG) and Chimal MHP .

Table 2: Simulation Data for Local Distribution grid and Chimpal MHP
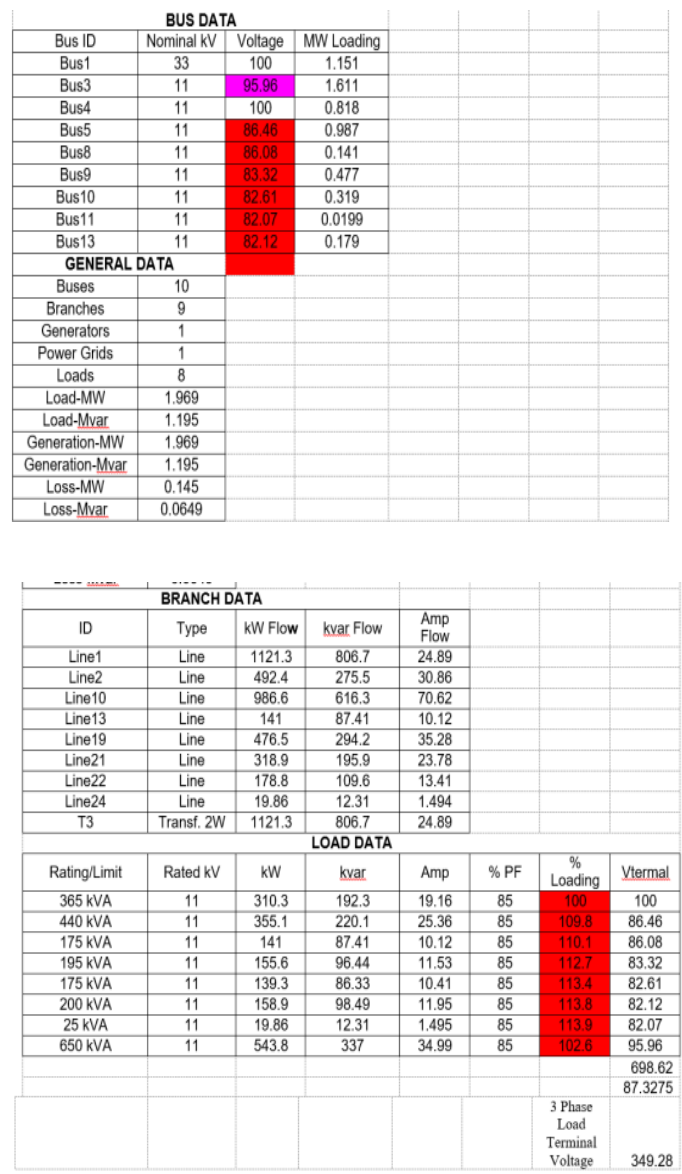

\begin{tabular}{|c|c|c|c|c|c|c||}
\multicolumn{7}{|c|}{ SOURCE DATA } \\
\hline Terminal Bus & Rating/Limit & $\begin{array}{c}\text { Rated } \\
\text { kV }\end{array}$ & MW & Mvar & Amp & $\%$ PF \\
\hline Bus4 & 0.09 MW & 11 & 0.818 & 0.483 & 49.85 & 86.12 \\
\hline Bus1 & 30 MVA & 33 & 1.151 & 0.712 & 23.68 & 85.02 \\
\hline
\end{tabular}

c) In Case-3, simulation is done with Local Distribution grid and $500 \mathrm{KW}$ MHP

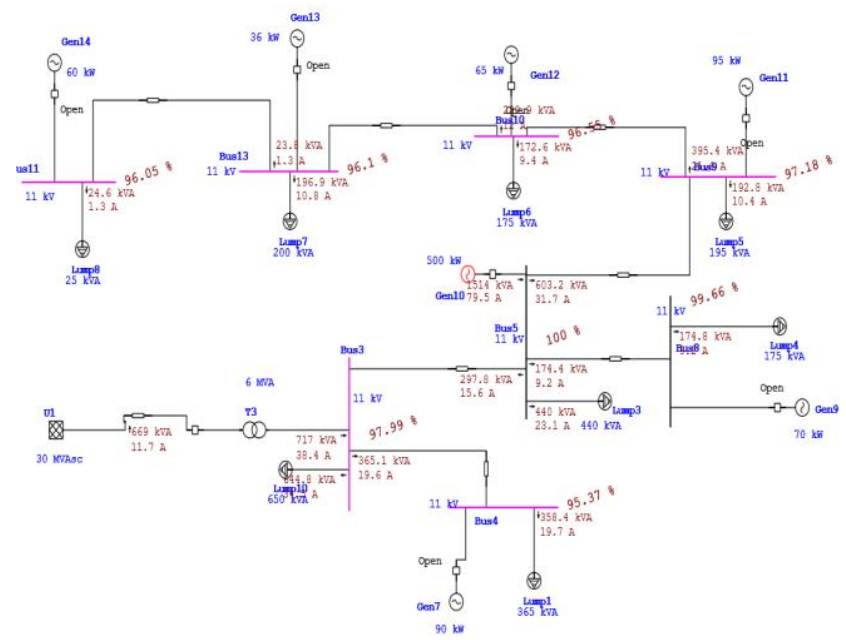

Figure 6: Load flow analysis for Local Distribution Grid (LDG) and $500 \mathrm{KW}$ MHP Plant.

Table 3: Simulation Data for Local Distribution Grid (LDG) and $500 \mathrm{KW}$ MHP Plant.

\begin{tabular}{|c|c|c|c|c|c|c|c|c|}
\hline \multicolumn{9}{|c|}{ LOAD DATA } \\
\hline ID & Rating/Limit & Rated kV & $\mathrm{kW}$ & kvar & Amp & $\%$ PF & $\begin{array}{c}\% \\
\text { Loading }\end{array}$ & Vtern \\
\hline Lump1 & $365 \mathrm{kVA}$ & 11 & 304.6 & 188.8 & \begin{tabular}{|l|}
19.72 \\
\end{tabular} & 85 & 103 & 95.3 \\
\hline Lump3 & $440 \mathrm{kVA}$ & 11 & 374 & 231.8 & 23.09 & 85 & 100 & 100 \\
\hline Lump4 & $175 \mathrm{kVA}$ & 11 & 148.5 & 92.06 & 9.204 & 85 & 100.2 & 99.6 \\
\hline Lump5 & $195 \mathrm{kVA}$ & 11 & 163.9 & 101.6 & 10.41 & 85 & 101.8 & 97.1 \\
\hline Lump6 & $175 \mathrm{kVA}$ & 11 & 146.7 & 90.94 & 9.385 & 85 & 102.2 & 96.5 \\
\hline Lump7 & $200 \mathrm{kVA}$ & 11 & 167.4 & 103.7 & 10.76 & 85 & 102.5 & 96.1 \\
\hline Lump8 & $25 \mathrm{kVA}$ & 11 & 20.92 & 12.97 & 1.345 & 85 & 102.5 & 96.0 \\
\hline Lump10 & $650 \mathrm{kVA}$ & 11 & 548.1 & 339.7 & 34.54 & 85 & 101.2 & 97.9 \\
\hline & & & & & & & & 778. \\
\hline & & & & & & & & 97.3 \\
\hline & & & & & & $\begin{array}{l}3 \text { Phase Load } \\
\text { Terminal Voltage }\end{array}$ & & 389. \\
\hline \multicolumn{8}{|c|}{ SOURCE DATA } & \\
\hline ID & Terminal & Ratinglimit & Rated kV & MW & Mvar & Amp & $\% \mathrm{PF}$ & \\
\hline
\end{tabular}

\begin{tabular}{|c|c|c|c|c|c|c|c|c|}
\hline & Bus & & & & & & & \\
\hline Gen10 & Bus5 & $0.5 \mathrm{MW}$ & 11 & 1.302 & 0.773 & 79.48 & 85.99 & \\
\hline U1 & Bus1 & $30 \mathrm{MVA}$ & 33 & 0.604 & 0.288 & 11.7 & 90.29 & \\
\hline \multicolumn{5}{|c|}{ BRANCH DATA } & & \multicolumn{2}{|c|}{ GENERAL DATA } & \\
\hline ID & Type & kW Flow & kvar Flow & $\begin{array}{l}\text { Amp } \\
\text { Flow }\end{array}$ & & Buses & 10 & \\
\hline Line1 & Line & 596.6 & 405.1 & 12.8 & & Branches & 9 & \\
\hline Line2 & Line & 304.6 & 188.8 & 19.72 & & Generators & 1 & \\
\hline Line10 & Line & 263.1 & 132.4 & 15.78 & & Power Grids & 1 & \\
\hline Line13 & Line & 148.5 & 92.06 & 9.204 & & Loads & 8 & \\
\hline Line19 & Line & 501.3 & 307.8 & 31.77 & & & & \\
\hline Line21 & Line & 335.7 & 205.3 & 21.39 & & Load-MW & 1.906 & \\
\hline Line22 & Line & 188.3 & 115.1 & 12.06 & & Load-Mvar & 1.06 & \\
\hline Line24 & Line & 20.92 & 12.97 & 1.345 & & Generation-MW & 1.906 & \\
\hline T3 & Transf. $2 \mathrm{~W}$ & 596.6 & 405.1 & 12.8 & & Generation-Mvar & 1.06 & \\
\hline & & & & & & Loss-MW & 0.0319 & \\
\hline \multicolumn{4}{|c|}{ BUS DATA } & & & Loss-Mvar & -0.101 & \\
\hline Bus ID & Nominal kV & Voltage & $\begin{array}{l}\text { MW } \\
\text { Loading }\end{array}$ & & & & & \\
\hline Bus1 & 33 & 100 & 0.604 & & & & & \\
\hline Bus3 & 11 & 97.99 & 0.859 & & & & & \\
\hline Bus4 & 11 & 95.37 & 0.305 & & & & & \\
\hline Bus5 & 11 & 100 & 1.302 & & & & & \\
\hline Bus8 & 11 & 99.66 & 0.149 & & & & & \\
\hline Bus9 & 11 & 97.18 & 0.501 & & & & & \\
\hline Bus10 & 11 & 96.55 & 0.336 & & & & & \\
\hline Bus11 & 11 & 96.05 & 0.0209 & & & & & \\
\hline Bus13 & 11 & 96.1 & 0.188 & & & & & \\
\hline
\end{tabular}


d) In Case-4, simulation is done with Local Distribution grid and Without $500 \mathrm{KW}$ MHP Plant .

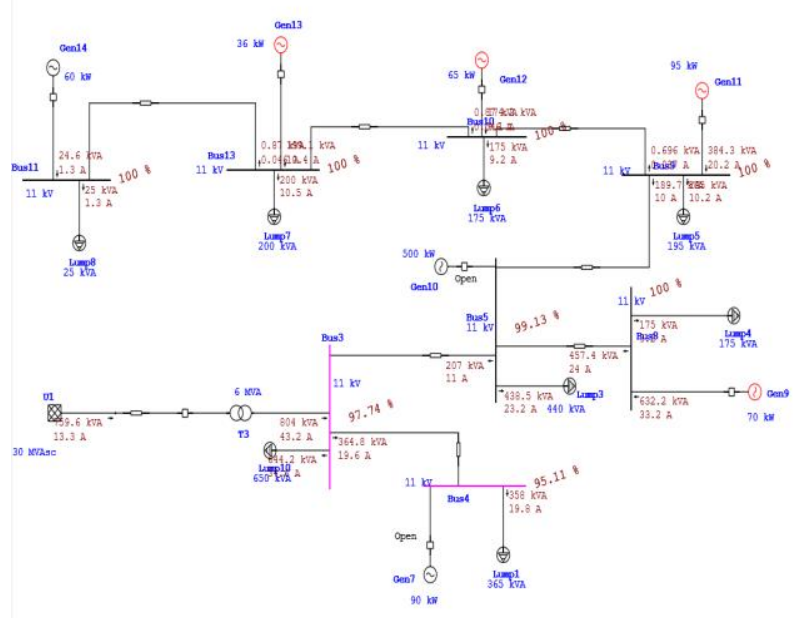

Figure 7: Load flow analysis for Local Distribution Grid (LDG) and Without 500 KW MHP Plant .

Table 4: Simulation for mini grid and Chimpal MHP

\begin{tabular}{|c|c|c|c|c|c|c|c|c|}
\hline \multicolumn{9}{|c|}{ LOAD DATA } \\
\hline ID & Rating/Limit & Rated kV & $\mathrm{kW}$ & kvar & Amp & $\% \mathrm{PF}$ & $\begin{array}{c}\% \\
\text { Loading }\end{array}$ & Vtel \\
\hline Lump1 & $365 \mathrm{kVA}$ & 11 & 304.3 & 188.6 & 19.76 & 85 & 103.1 & 95 \\
\hline Lump3 & $440 \mathrm{kVA}$ & 11 & 372.7 & 231 & 23.22 & 85 & 100.5 & 99 \\
\hline Lump4 & $175 \mathrm{kVA}$ & 11 & 148.8 & 92.19 & 9.185 & 85 & 100 & 11 \\
\hline Lump5 & $195 \mathrm{kVA}$ & 11 & 165.8 & 102.7 & 10.23 & 85 & 100 & 11 \\
\hline Lump6 & $175 \mathrm{kVA}$ & 11 & 148.8 & 92.19 & 9.185 & 85 & 100 & 11 \\
\hline Lump7 & $200 \mathrm{kVA}$ & 11 & 170 & 105.4 & 10.5 & 85 & 100 & 11 \\
\hline Lump8 & $25 \mathrm{kVA}$ & 11 & 21.25 & 13.17 & 1.312 & 85 & 100 & 11 \\
\hline Lump10 & $650 \mathrm{kVA}$ & 11 & 547.6 & 339.3 & 34.59 & 85 & 101.4 & 97 \\
\hline & & & & & & & & 791 \\
\hline & & & & & & & & 98 \\
\hline & & & & & & $\begin{array}{l}3 \text { Phase Load } \\
\text { Terminal Voltage }\end{array}$ & & 395 \\
\hline \multicolumn{7}{|c|}{ SOURCE DATA } & & \\
\hline ID & $\begin{array}{c}\text { Terminal } \\
\text { Bus }\end{array}$ & Rating/Limit & Rated kV & MW & Mvar & Amp & & \\
\hline Gen9 & Bus8 & $0.07 \mathrm{MW}$ & 11 & 0.548 & 0.316 & 33.18 & & \\
\hline Gen11 & Bus9 & $0.095 \mathrm{MW}$ & 11 & 0.332 & 0.194 & 20.17 & & \\
\hline Gen12 & Bus10 & $0.065 \mathrm{MW}$ & 11 & 0.149 & 0.0906 & 9.142 & & \\
\hline Gen13 & Bus13 & $0.036 \mathrm{MW}$ & 11 & 0.17 & 0.104 & 10.45 & & \\
\hline Gen14 & Bus11 & $0.06 \mathrm{MW}$ & 11 & 0.0213 & 0.0123 & 1.289 & & \\
\hline U1 & Bus1 & $30 \mathrm{MVA}$ & 33 & 0.682 & 0.335 & 13.29 & & \\
\hline
\end{tabular}

\begin{tabular}{|c|c|c|c|c|c|c|}
\hline \multicolumn{5}{|c|}{ BRANCH DATA } & \multicolumn{2}{|c|}{ GENERAL DATA } \\
\hline ID & Type & kW Flow & kvar Flow & $\begin{array}{l}\text { Amp } \\
\text { Flow }\end{array}$ & Buses & 10 \\
\hline Line1 & Line & 672.3 & 450.3 & 14.39 & Branches & 9 \\
\hline Line2 & Line & 304.3 & 188.6 & 19.76 & Generators & 5 \\
\hline Line10 & Line & 186.8 & 88.41 & 11.1 & Power Grids & 1 \\
\hline Line13 & Line & 396.3 & 221.9 & 24.05 & Loads & 8 \\
\hline Line19 & Line & 165.1 & 94.14 & 10.06 & Load-MW & 1.901 \\
\hline Line21 & Line & 0 & -0.696 & 0.037 & Load-Mvar & 1.051 \\
\hline Line22 & Line & 0 & -0.87 & 0.046 & Generation-MW & 1.901 \\
\hline Line24 & Line & 0 & -0.87 & 0.046 & Generation-Mvar & 1.051 \\
\hline \multirow[t]{2}{*}{ T3 } & Transf. 2W & 672.3 & 450.3 & 14.39 & Loss-MW & 0.0223 \\
\hline & & & & & Loss-Mvar & -0.114 \\
\hline \multicolumn{4}{|c|}{ BUS DATA } & & & \\
\hline Bus ID & Nominal kV & Voltage & $\begin{array}{c}\text { MW } \\
\text { Loading }\end{array}$ & & & \\
\hline Bus1 & 33 & 100 & 0.682 & & & \\
\hline Bus3 & 11 & 97.74 & 0.858 & & & \\
\hline Bus4 & 11 & 95.11 & 0.304 & & & \\
\hline Bus5 & 11 & 99.13 & 0.561 & & & \\
\hline Bus8 & 11 & 100 & 0.548 & & & \\
\hline Bus9 & 11 & 100 & 0.332 & & & \\
\hline Bus10 & 11 & 100 & 0.149 & & & \\
\hline Bus11 & 11 & 100 & 0.0213 & & & \\
\hline
\end{tabular}

e) In Case-5, simulation is done with mini grid and Chimal plant (90 KW).

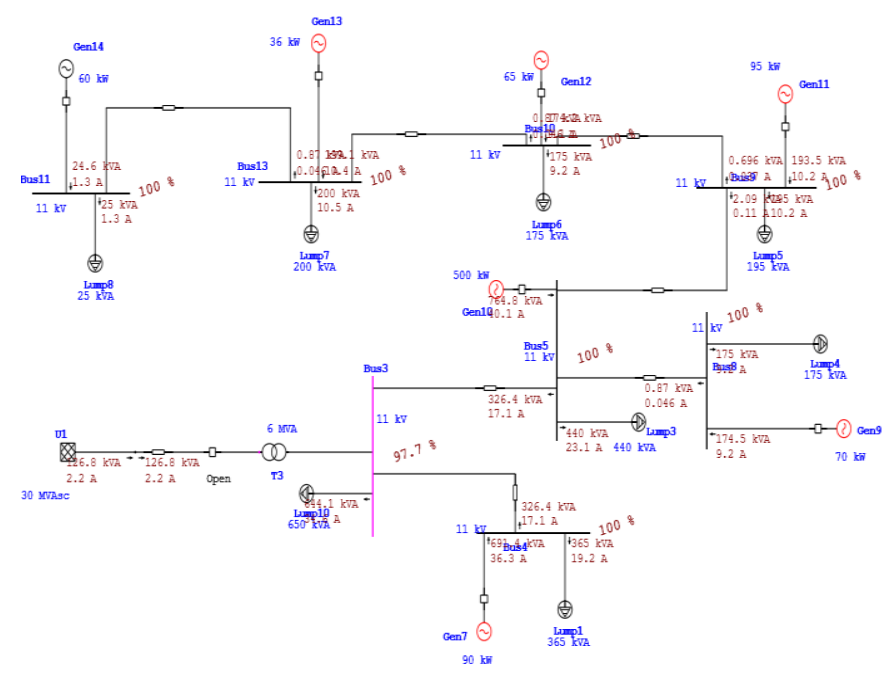

Figure 8: Load flow analysis for Mini-grid and Chimal MHP $(90 \mathrm{KW})$.

Table 5: Simulation Data for mini grid and Chimpal MHP Plant

\begin{tabular}{|c|c|c|c|c|c|c|c|c|}
\hline \multicolumn{9}{|c|}{ LOAD DATA } \\
\hline ID & Rating/Limit & Rated kV & $\mathrm{kW}$ & kvar & Amp & $\% \mathrm{PF}$ & $\begin{array}{c}\% \\
\text { Loading }\end{array}$ & Vterr \\
\hline Lump1 & $365 \mathrm{kVA}$ & 11 & 310.3 & 192.3 & 19.16 & 85 & 100 & 100 \\
\hline Lump3 & $440 \mathrm{kVA}$ & 11 & 374 & 231.8 & 23.09 & 85 & 100 & 100 \\
\hline Lump4 & $175 \mathrm{kVA}$ & 11 & 148.8 & 92.19 & 9.185 & 85 & 100 & 100 \\
\hline Lump5 & $195 \mathrm{kVA}$ & 11 & 165.8 & 102.7 & 10.23 & 85 & 100 & 100 \\
\hline Lump6 & $175 \mathrm{kVA}$ & 11 & 148.8 & 92.19 & 9.185 & 85 & 100 & 100 \\
\hline Lump7 & $200 \mathrm{kVA}$ & 11 & 170 & 105.4 & 10.5 & 85 & 100 & 100 \\
\hline Lump8 & $25 \mathrm{kVA}$ & 11 & 21.25 & 13.17 & 1.312 & 85 & 100 & 100 \\
\hline Lump10 & $650 \mathrm{kVA}$ & 11 & 547.5 & 339.3 & 34.6 & 85 & 101.4 & 97.7 \\
\hline & & & & & & & & $797 . i$ \\
\hline & & & & & & & & 99.7 \\
\hline & & & & & & $\begin{array}{l}3 \text { Phase Load } \\
\text { Terminal Voltage }\end{array}$ & & 398.8 \\
\hline \multicolumn{9}{|c|}{ SOURCE DATA } \\
\hline ID & $\begin{array}{l}\text { Terminal } \\
\text { Bus }\end{array}$ & Rating/Limit & Rated kV & MW & Mvar & Amp & $\% \mathrm{PF}$ & \\
\hline Gen7 & Bus4 & $0.09 \mathrm{MW}$ & 11 & 0.589 & 0.362 & 36.29 & 85.16 & \\
\hline Gen9 & Bus8 & $0.07 \mathrm{MW}$ & 11 & 0.149 & 0.0913 & 9.161 & 85.22 & \\
\hline Gen10 & Bus5 & $0.5 \mathrm{MW}$ & 11 & 0.653 & 0.399 & 40.14 & 85.32 & \\
\hline Gen11 & Bus9 & $0.095 \mathrm{MW}$ & 11 & 0.166 & 0.0999 & 10.16 & 85.64 & \\
\hline
\end{tabular}

\begin{tabular}{|c|c|c|c|c|c|c|c|}
\hline Gen12 & Bus10 & $0.065 \mathrm{MW}$ & 11 & 0.149 & \begin{tabular}{|l|l|}
0.0906 \\
\end{tabular} & 9.142 & 85.4 \\
\hline Gen13 & Bus13 & $0.036 \mathrm{MW}$ & 11 & 0.17 & 0.104 & 10.45 & 85.39 \\
\hline Gen14 & Bus11 & $0.06 \mathrm{MW}$ & 11 & 0.0213 & 0.0123 & 1.289 & 86.55 \\
\hline U1 & Bus1 & $30 \mathrm{MVA}$ & 33 & 0.0001 & -0.127 & 2.218 & -0.05 \\
\hline \multicolumn{5}{|c|}{ BRANCH DATA } & & \multicolumn{2}{|c|}{ GENERAL DATA } \\
\hline ID & Type & kW Flow & kvar Flow & $\begin{array}{l}\text { Amp } \\
\text { Flow }\end{array}$ & & Buses & 11 \\
\hline Line1 & Line & 0.061 & -126.8 & 2.218 & & Branches & 9 \\
\hline Line2 & Line & 273.7 & 169.7 & 17.3 & & Generators & 7 \\
\hline Line10 & Line & 273.7 & 169.7 & 17.3 & & Power Grids & 1 \\
\hline Line13 & Line & 0 & -0.87 & 0.046 & & Loads & 8 \\
\hline Line19 & Line & 0 & -2.09 & 0.11 & & & \\
\hline Line21 & Line & 0 & -0.696 & 0.037 & & Load-MW & 1.896 \\
\hline Line22 & Line & 0 & -0.87 & 0.046 & & Load-Mvar & 1.032 \\
\hline Line24 & Line & 0 & -0.87 & 0.046 & & Generation-MW & 1.896 \\
\hline \multirow[t]{2}{*}{ T3 } & Transf. 2W & 0 & 0 & 0 & & Generation-Mvar & 1.032 \\
\hline & & & & & & Loss-MW & 0.0097 \\
\hline \multicolumn{4}{|c|}{ BUS DATA } & & & Loss-Mvar & -0.137 \\
\hline Bus ID & Nominal kV & Voltage & $\begin{array}{c}\text { MW } \\
\text { Loading }\end{array}$ & & & & \\
\hline Bus1 & 33 & 100 & 0.0001 & & & & \\
\hline Bus3 & 11 & 97.7 & 0.547 & & & & \\
\hline Bus4 & 11 & 100 & 0.589 & & & & \\
\hline Bus5 & 11 & 100 & 0.653 & & & & \\
\hline Bus8 & 11 & 100 & 0.149 & & & & \\
\hline Bus9 & 11 & 100 & 0.166 & & & & \\
\hline Bus10 & 11 & 100 & 0.149 & & & & \\
\hline Bus11 & 11 & 100 & 0.0213 & & & & \\
\hline Bus13 & 11 & 100 & 0.17 & & & & \\
\hline
\end{tabular}


f) InCase-6, simulation is done with grid integration of Chimal plant (90 KW), mini grid and Local Distribution grid .

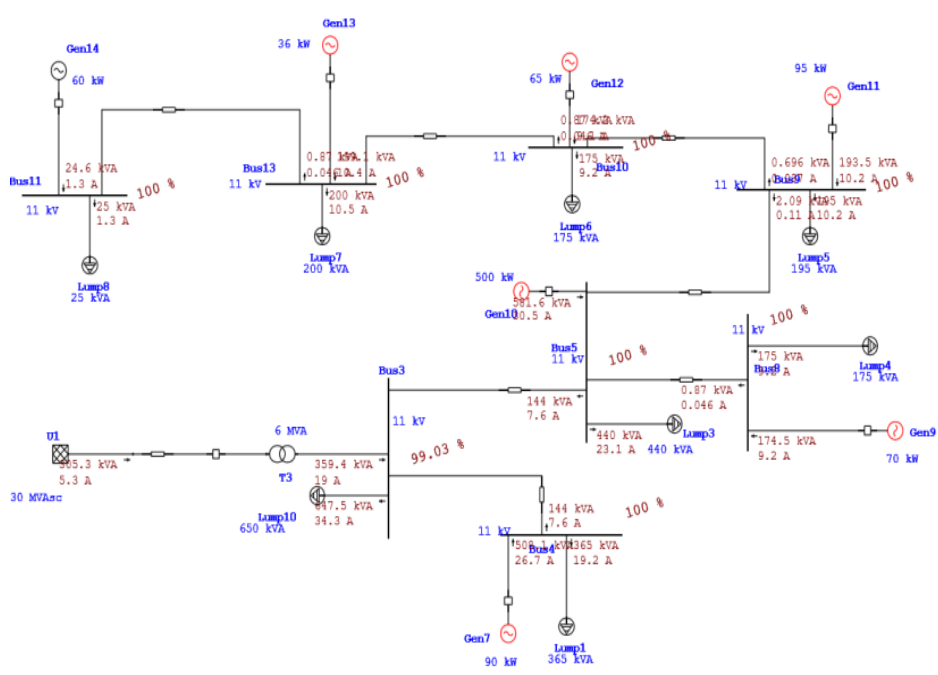

Figure 9: Load flow analysis for Local Distribution Grid (LDG),Mini grid and Chimal MHP Plant.

Table 6: Simulation Data for mini grid, Chimal MHP and Local Distribution grid .

\begin{tabular}{|c|c|c|c|c|c|c|c|c|}
\hline \multicolumn{9}{|c|}{ LOAD DATA } \\
\hline ID & Rating/Limit & Rated kV & $\mathrm{kW}$ & kvar & Amp & $\% \mathrm{PF}$ & $\begin{array}{c}\% \\
\text { Loading }\end{array}$ & Vtermal \\
\hline Lump1 & $365 \mathrm{kVA}$ & 11 & 310.3 & 192.3 & 19.16 & 85 & 100 & 100 \\
\hline Lump3 & $440 \mathrm{kVA}$ & 11 & 374 & 231.8 & 23.09 & 85 & 100 & 100 \\
\hline Lump4 & $175 \mathrm{kVA}$ & 11 & 148.8 & 92.19 & 9.185 & 85 & 100 & 100 \\
\hline Lump5 & $195 \mathrm{kVA}$ & 11 & 165.8 & 102.7 & 10.23 & 85 & 100 & 100 \\
\hline Lump6 & $175 \mathrm{kVA}$ & 11 & 148.8 & 92.19 & 9.185 & 85 & 100 & 100 \\
\hline Lump7 & $200 \mathrm{kVA}$ & 11 & 170 & 105.4 & 10.5 & 85 & 100 & 100 \\
\hline Lump8 & $25 \mathrm{kVA}$ & 11 & 21.25 & 13.17 & 1.312 & 85 & 100 & 100 \\
\hline Lump10 & $650 \mathrm{kVA}$ & 11 & 550.4 & 341.1 & 34.32 & 85 & 100.6 & 99.03 \\
\hline & & & & & & & & 799 \\
\hline & & & & & & & & 99.9 \\
\hline & & & & & & & & 399 \\
\hline \multicolumn{9}{|c|}{ SOURCE DATA } \\
\hline ID & $\begin{array}{c}\text { Terminal } \\
\text { Bus }\end{array}$ & Rating/Limit & Rated kV & MW & Mvar. & Amp & $\% \mathrm{PF}$ & \\
\hline Gen7 & Bus4 & $0.09 \mathrm{MW}$ & 11 & 0.441 & 0.252 & 26.67 & 86.85 & \\
\hline Gen9 & Bus8 & $0.07 \mathrm{MW}$ & 11 & 0.149 & 0.0913 & 9.161 & 85.22 & \\
\hline Gen10 & Bus5 & $0.5 \mathrm{MW}$ & 11 & 0.505 & 0.288 & 30.53 & 86.84 & \\
\hline Gen11 & Bus9 & $0.095 \mathrm{MW}$ & 11 & 0.166 & 0.0999 & 10.16 & 85.64 & \\
\hline Gen12 & Bus10 & $0.065 \mathrm{MW}$ & 11 & 0.149 & 0.0906 & 9.142 & 85.4 & \\
\hline Gen13 & Bus13 & $0.036 \mathrm{MW}$ & 11 & 0.17 & 0.104 & 10.45 & 85.39 & \\
\hline Gen14 & Bus11 & $0.06 \mathrm{MW}$ & 11 & 0.0213 & 0.0123 & 1.289 & 86.55 & \\
\hline U1 & Bus1 & 30 MVA & 33 & 0.292 & 0.0894 & 5.342 & 95.61 & \\
\hline
\end{tabular}

\begin{tabular}{|c|c|c|c|c|c|c|}
\hline \multicolumn{5}{|c|}{ BRANCH DATA } & \multicolumn{2}{|c|}{ GENERAL DATA } \\
\hline ID & Type & kW Flow & kvar Flow & $\begin{array}{l}\text { Amp } \\
\text { Flow }\end{array}$ & Buses & 10 \\
\hline Line1 & Line & 290.3 & 213.7 & 6.349 & Branches & 9 \\
\hline Line2 & Line & 130.1 & 64.48 & 7.697 & Generators & 7 \\
\hline Line10 & Line & 130.1 & 64.48 & 7.697 & Power Grids & 1 \\
\hline Line13 & Line & 0 & -0.87 & 0.046 & Loads & 8 \\
\hline Line19 & Line & 0 & -2.09 & 0.11 & & \\
\hline Line21 & Line & 0 & -0.696 & 0.037 & Load-MW & 1.893 \\
\hline Line22 & Line & 0 & -0.87 & 0.046 & Load-Mvar & 1.027 \\
\hline Line24 & Line & 0 & -0.87 & 0.046 & \begin{tabular}{|l|} 
Generation-MW \\
\end{tabular} & 1.893 \\
\hline T3 & Transf. 2W & 290.3 & 213.7 & 6.349 & \begin{tabular}{|c|}
$\begin{array}{c}\text { Generation- } \\
\text { Mvar }\end{array}$ \\
\end{tabular} & 1.027 \\
\hline \multirow{2}{*}{\multicolumn{5}{|c|}{ BUS DATA }} & Loss-MW & 0.0037 \\
\hline & & & & & Loss-Mvar & -0.143 \\
\hline Bus ID & Nominal kV & Voltage & $\begin{array}{l}\text { MW } \\
\text { Loading }\end{array}$ & & & \\
\hline Bus1 & 33 & 100 & 0.292 & & & \\
\hline Bus3 & 11 & 99.03 & 0.55 & & & \\
\hline Bus4 & 11 & 100 & 0.441 & & & \\
\hline Bus5 & 11 & 100 & 0.505 & & & \\
\hline Bus8 & 11 & 100 & 0.149 & & & \\
\hline
\end{tabular}

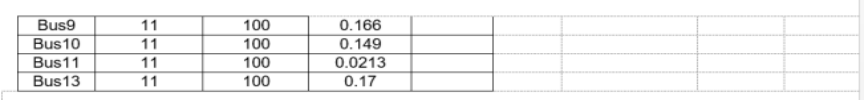

g) InCase-7, simulation is done only for mini grid .

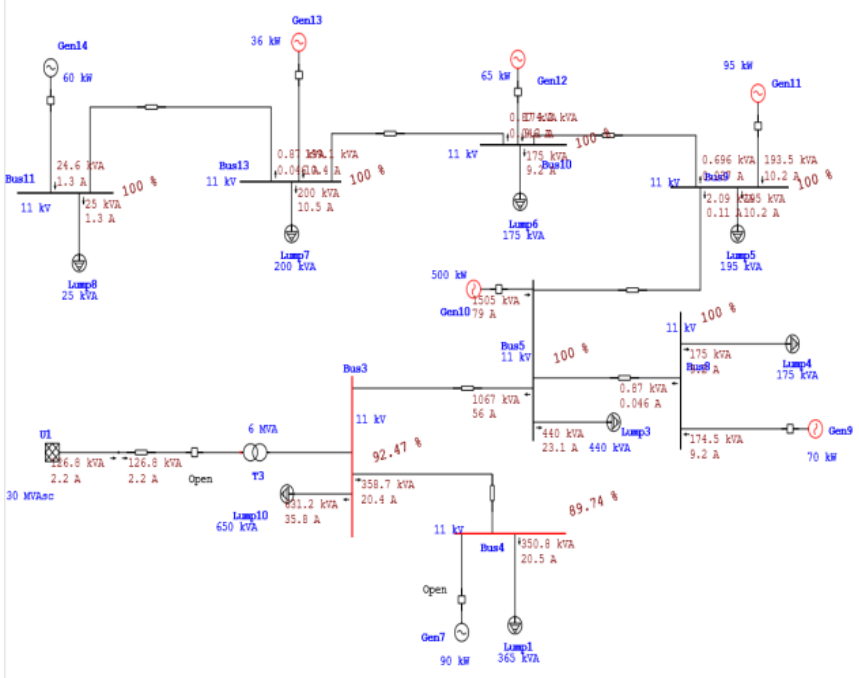

Figure 10: Simulation Diagram for Mini Grid Only.

Table 7: Simulation for mini grid Only.

\begin{tabular}{|c|c|c|c|c|c|c|c|c|}
\hline \multicolumn{9}{|c|}{ LOAD DATA } \\
\hline ID & Rating/Limit & Rated kV & $\mathrm{kW}$ & kvar & Amp & $\% \mathrm{PF}$ & $\begin{array}{c}\% \\
\text { Loading }\end{array}$ & Vterm \\
\hline Lump1 & $365 \mathrm{kVA}$ & 11 & 298.2 & 184.8 & 20.52 & 85 & 107.1 & 89.74 \\
\hline Lump3 & $440 \mathrm{kVA}$ & 11 & 374 & 231.8 & 23.09 & 85 & 100 & 100 \\
\hline Lump4 & $175 \mathrm{kVA}$ & 11 & 148.8 & 92.19 & 9.185 & 85 & 100 & 100 \\
\hline Lump5 & $195 \mathrm{kVA}$ & 11 & 165.8 & 102.7 & 10.23 & 85 & 100 & 100 \\
\hline Lump6 & $175 \mathrm{kVA}$ & 11 & 148.8 & 92.19 & 9.185 & 85 & 100 & 100 \\
\hline Lump7 & $200 \mathrm{kVA}$ & 11 & 170 & 105.4 & 10.5 & 85 & 100 & 100 \\
\hline Lump8 & $25 \mathrm{kVA}$ & 11 & 21.25 & 13.17 & 1.312 & 85 & 100 & 100 \\
\hline Lump10 & $650 \mathrm{kVA}$ & 11 & 536.5 & 332.5 & 35.82 & 85 & 105 & 92.4 \\
\hline & & & & & & & & 782.2 \\
\hline & & & & & & & & 97.77 \\
\hline & & & & & & $\begin{array}{l}3 \text { Phase Load } \\
\text { Terminal Voltage }\end{array}$ & & 391.0 \\
\hline \multicolumn{9}{|c|}{ SOURCE DATA } \\
\hline ID & $\begin{array}{l}\text { Terminal } \\
\text { Bus }\end{array}$ & Rating/Limit & Rated kV & MW & Mvar & Amp & $\% \mathrm{PF}$ & \\
\hline Gen9 & Bus8 & $0.07 \mathrm{MW}$ & 11 & 0.149 & 0.0913 & 9.161 & 85.22 & \\
\hline
\end{tabular}

\begin{tabular}{|c|c|c|c|c|c|c|c|}
\hline Gen12 & Bus10 & $0.065 \mathrm{MW}$ & 11 & 0.149 & 0.0906 & 9.142 & 85.4 \\
\hline Gen13 & Bus13 & $0.036 \mathrm{MW}$ & 11 & 0.17 & 0.104 & 10.45 & 85.39 \\
\hline Gen14 & Bus11 & $0.06 \mathrm{MW}$ & 11 & 0.0213 & 0.0123 & 1.289 & 86.55 \\
\hline U1 & Bus1 & 30 MVA & 33 & 0.0001 & \begin{tabular}{|c|}
-0.127 \\
\end{tabular} & 2.218 & -0.05 \\
\hline \multicolumn{5}{|c|}{ BRANCH DATA } & & \multicolumn{2}{|c|}{ GENERAL DATA } \\
\hline ID & Type & kW Flow & kvar Flow & $\begin{array}{l}\text { Amp } \\
\text { Flow }\end{array}$ & & Buses & 11 \\
\hline Line1 & Line & 0.061 & -126.8 & 2.218 & & Branches & 9 \\
\hline Line2 & $\operatorname{Lin} \theta$ & 298.2 & 184.8 & 20.52 & & Generators & 6 \\
\hline Line10 & Line & 841.4 & 521.3 & 56.18 & & Power Grids & 1 \\
\hline Line13 & Line & 0 & .0 .87 & 0.046 & & Loads & 8 \\
\hline Line19 & Line & 0 & -2.09 & 0.11 & & Load-MW & 1.921 \\
\hline Line21 & Line & 0 & .0 .696 & 0.037 & & Load-Mvar & 1.085 \\
\hline Line22 & Line & 0 & .0 .87 & 0.046 & & Generation-MW & 1.921 \\
\hline Line24 & Line & 0 & -0.87 & 0.046 & & $\begin{array}{c}\text { Generation- } \\
\text { Mvar }\end{array}$ & 1.085 \\
\hline T3 & Transf. 2W & 0 & 0 & 0 & & Loss-MW & 0.0578 \\
\hline & & & & & & Loss-Mvar & -0.0696 \\
\hline \multicolumn{4}{|c|}{ BUS DATA } & & & & \\
\hline Bus ID & Nominal kV & Voltage & $\begin{array}{c}\text { MW } \\
\text { Loading }\end{array}$ & & & & \\
\hline Bus1 & 33 & 100 & 0.0001 & & & & \\
\hline Bus3 & 11 & 92.47 & 0.841 & & & & \\
\hline Bus4 & 11 & 89.74 & 0.298 & & & & \\
\hline Bus5 & 11 & 100 & 1.266 & & & & \\
\hline Bus8 & 11 & 100 & 0.149 & & & & \\
\hline Bus9 & 11 & 100 & 0.166 & & & & \\
\hline Bus10 & 11 & 100 & 0.149 & & & & \\
\hline Bus11 & 11 & 100 & 0.0213 & & & & \\
\hline Bus13 & 11 & 100 & 0.17 & & & & \\
\hline
\end{tabular}




\section{GRID IMPACT STUDY}

A technical evaluation is performed using ETAP to illustrate the viability of integration of MHP plant and mini grid with Local distribution grid.

\section{A. Load Flow Analysis}

For load flow analysis, the Taplejung substation distribution network and the existing electrical network at Mini grid together with digitized on ETAP software as per the information collected and then simulated for the corresponding load. Amarpur substation $33 \mathrm{kV}$ bus is considered as slack bus for load flow analysis.

With load flow analysis, the total losses on different Cases is analyzed under different cases and depicted in Figure 11. The total power loss of the system is maximum in case-1, which is around $206 \mathrm{KW}$, and the loss decreases as the power exported from mini grid increases. The losses on the system is minimum for case- 6 and it is around $3.7 \mathrm{KW}$. This is because the line loss is proportional to square of the current flowing through the line. Likewise the voltage drop in the line and terminal voltage at the different load point is observed and shown in Figure 12. It is seen that the voltage drop in the lines decreases and terminal voltage at different load points get improved as the surplus power is exported from mini grid and MHP plant.

\section{RESULTS AND DISCUSSION}

- Power Losses at different cases is presented in the diagrams below indicating reduction for the interconnection of mini grid with Local Distribution grid.

- The interconnection of mini grids with Local Distribution grid is seen to be significant in minimizing the distribution system losses in Taplejung Mini grid, hence, it shall greatly impart the benefits to the economy.

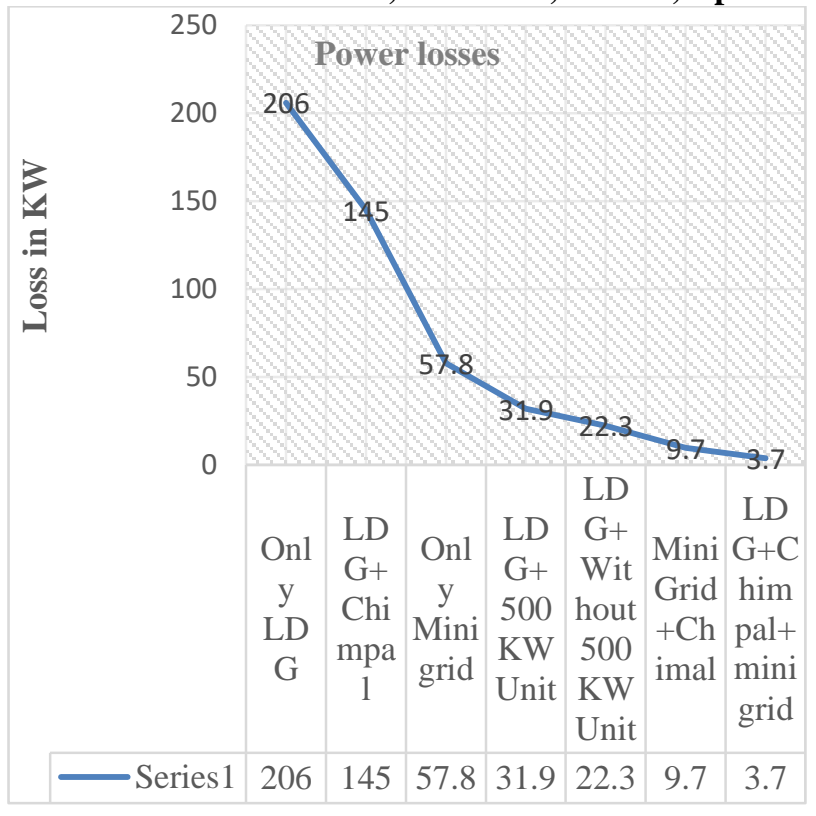

Figure 11: Power losses in system at various cases

The quality of electricity supply through combined system of interconnected mini grid \& LDG is found to be better examining the terminal voltage at equipment. The terminal voltage is found to be acceptable limits when the mini grid, MHP is connected with the LDG which can be well illustrated by the figure below.

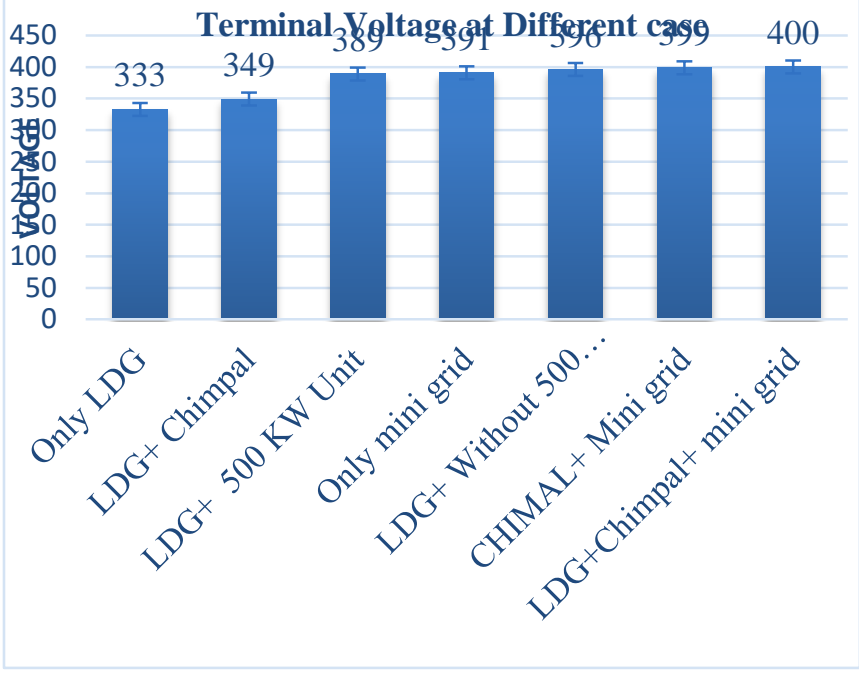

Figure 12: Terminal Voltage at various conditions

The terminal voltage at various loads modelled is plot graphically each point indicating percentage of terminal voltage received to that should have been received. The results of simulation shows that when the interconnected system is operated, loads receive terminal voltage at an acceptable level of supply. The cases LDG(NEA) supply only, LDG \& Chimpal show terminal voltage below standard limit at some loads while the cases interconnected Mini Grid-LDG-Chimpal, LDG-Mini Grid and Mini Grid-Chimpal show the supply at standard level of terminal voltage. 


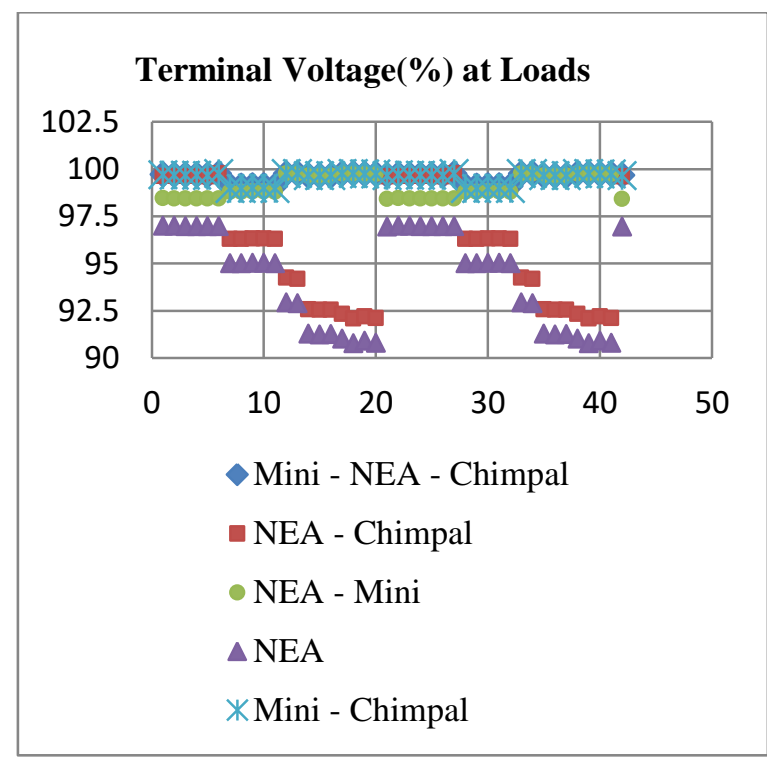

Figure 13: Terminal Voltage at various loads in different conditions of Operation

The voltage drop in the different case is observed and shown in figure 14 .From figure it is seen that the voltage drop decrease as the power is exported from mini grid and MHP Plant.

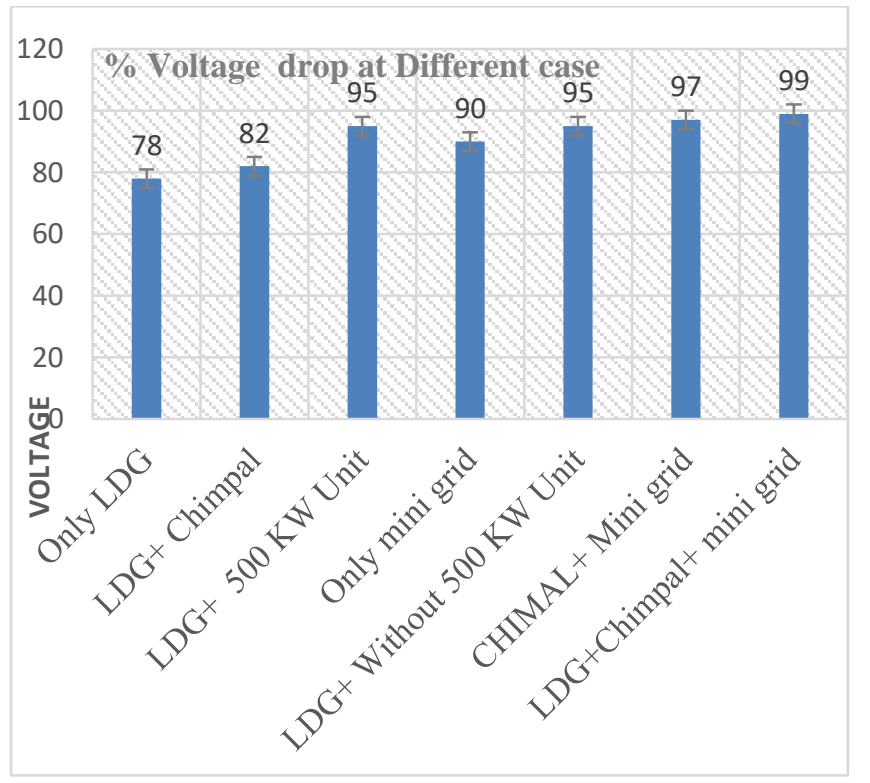

Figure 14: Percentage Voltage drop at different conditions of Operation

The KVA chart illustrates that the load supplied by LDG is greatly reduced when the mini grid \& isolated generator both are interconnected with the LDG indicating the economy of electric supply.

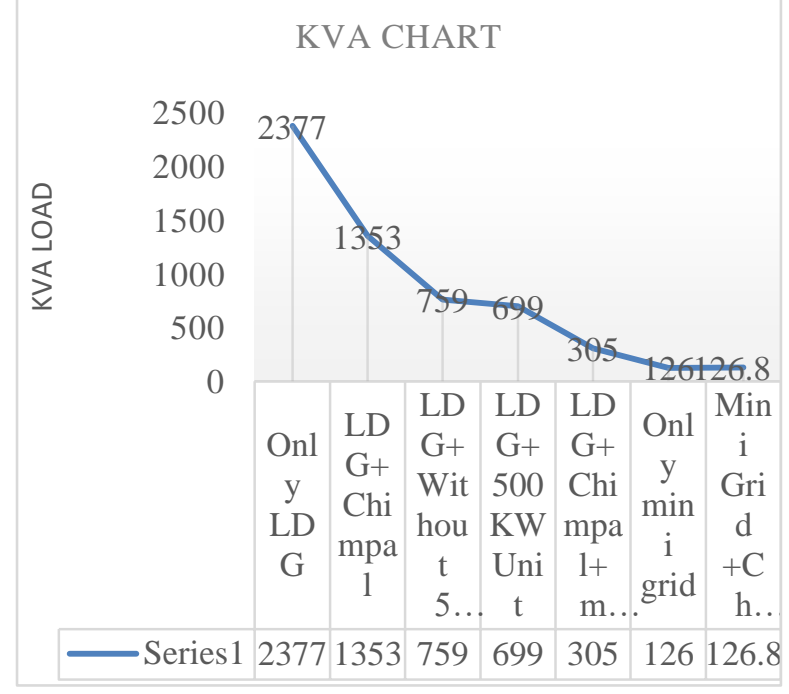

Figure 15: KVA Chart at various cases

The current chart illustrates the reduction in current flowing during the interconnected system operation, which eventually minimizes loss serving benefits to the system.

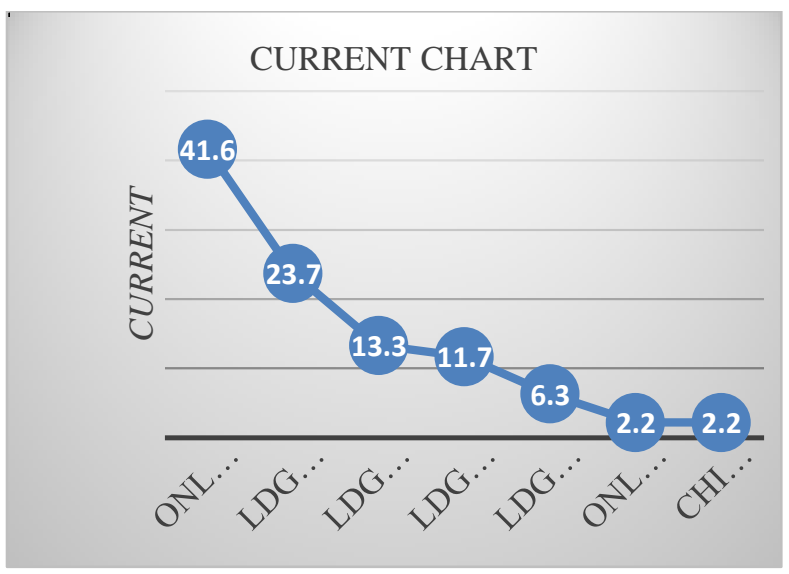

Figure 16: Current Chart at various Cases

\section{OPTIMIZATION OF DG}

To improve the voltage magnitudes and reduce power losses, Distributed Generators were placed optimally in the Network [10][12].

\section{A. Simulation Phase I: Power System without DG}

In Phase-I of optimization, the load flow analysis was executed on power system without being installing the DG so that the impact of power losses was studied. The simulation shows total losses of entire power system as shown in fig 4 and Table 1.

The overall system losses after execution of load flow analysis without DG were $206 \mathrm{KW}$. This is the huge power losses for any power system that must be minimized by insertion of distribution generation. We have selected DG with the rating of $826 \mathrm{KW}$. The DG must be placed in optimal location so that 
DG may be connected to the bus which gives lowest overall power system losses and improve in voltage profile.

\section{B. $\quad$ Simulation Phase II: Power System with DG}

Distributed generation having capacity $826 \mathrm{KW}$ is now connected to the power system and find out the effect of DG on the entire system. Bus-2 is connected to the main grid station so its voltage rating is $33 \mathrm{KV}$; while the remaining buses are rated with $11 \mathrm{KV}$.

\section{a) Power system when DG to Bus-3}

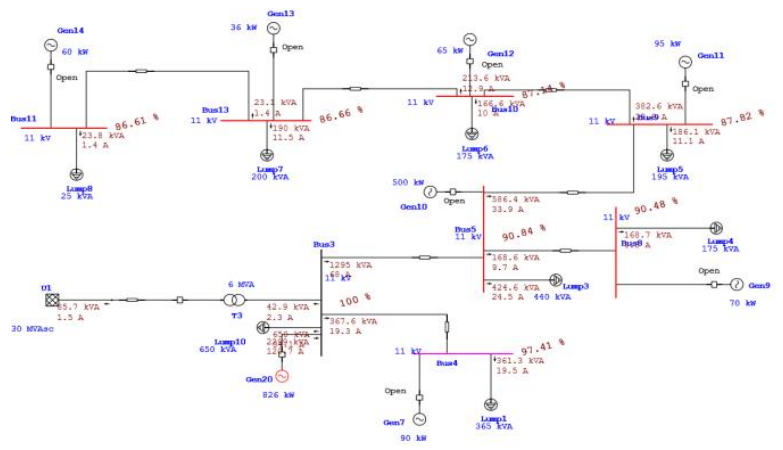

Figure 17: Load flow analysis of power system when DG to Bus 3

Table 8: Data of Load flow analysis of power system when DG to Bus 3.

\begin{tabular}{|c|c|c|c|c|c|c|c|c|}
\hline \multicolumn{9}{|c|}{ LOAD DATA } \\
\hline ID & Rating/Limit & Rated kV & $\mathrm{kW}$ & kvar & Amp & $\% \mathrm{PF}$ & $\begin{array}{c}\% \\
\text { Loading }\end{array}$ & Vtermi \\
\hline Lump1 & $365 \mathrm{kVA}$ & 11 & 307.1 & 190.3 & 19.47 & 85 & 101.6 & 97.41 \\
\hline Lump3 & $440 \mathrm{kVA}$ & 11 & 360.9 & 223.7 & 24.53 & 85 & 106.2 & 90.84 \\
\hline Lump4 & $175 \mathrm{kVA}$ & 11 & 143.4 & 88.84 & 9.783 & 85 & 106.5 & 90.48 \\
\hline Lump5 & $195 \mathrm{kVA}$ & 11 & 158.2 & 98.02 & 11.12 & 85 & 108.7 & 87.82 \\
\hline Lump6 & $175 \mathrm{kVA}$ & 11 & 141.6 & 87.75 & 10.03 & 85 & 109.2 & 87.14 \\
\hline $\begin{array}{l}\text { Lump7 } \\
\end{array}$ & $200 \mathrm{kVA}$ & 11 & 161.5 & 100.1 & 11.51 & 85 & 109.6 & 86.66 \\
\hline Lump8 & $25 \mathrm{kVA}$ & 11 & 20.19 & 12.51 & 1.439 & 85 & 109.7 & 86.61 \\
\hline Lump10 & $650 \mathrm{kVA}$ & 11 & 552.5 & 342.4 & 34.12 & 85 & 100 & 100 \\
\hline & & & & & & & & 726.96 \\
\hline & & & & & & & & 90.87 \\
\hline & & & & & & $\begin{array}{l}\text { 3Phase Load } \\
\text { Terminal Voltage }\end{array}$ & & $363.4 \varepsilon$ \\
\hline \multirow{2}{*}{\multicolumn{9}{|c|}{ SOURCE DATA }} \\
\hline & & & & & & & & \\
\hline ID & $\begin{array}{c}\text { Terminal } \\
\text { Bus }\end{array}$ & Rating/Limit & Rated kV & MW & Mvar & Amp & $\% \mathrm{PF}$ & \\
\hline Gen20 & Bus3 & $0.826 \mathrm{MW}$ & 11 & 1.952 & 1.214 & 120.7 & 84.92 & \\
\hline U1 & Bus1 & $30 \mathrm{MVA}$ & 33 & -0.0101 & 0.0851 & 1.499 & 11.83 & \\
\hline
\end{tabular}

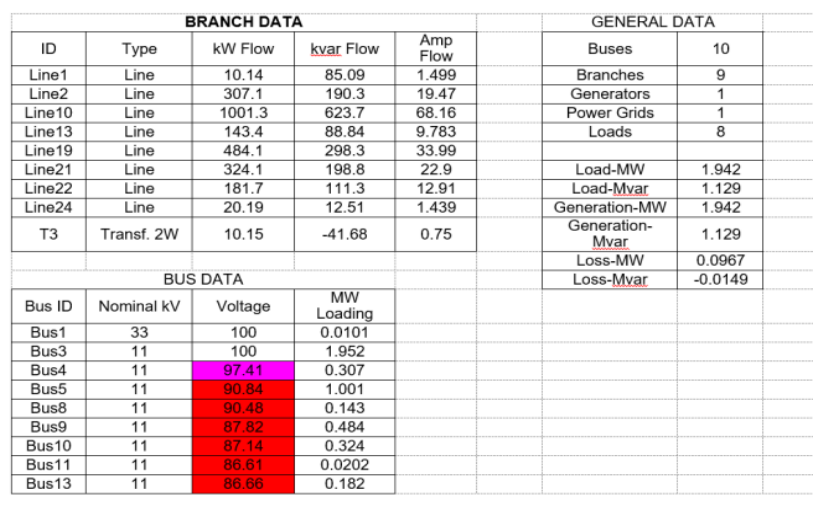

The Percentage voltage drop, load terminal voltage, KVA and current flowing at each bus is shown in Table 8 and Figure 17.
From table, it is clear that he overall system losses after execution of load flow analysis when DG to Bus 3 was 96.7 $\mathrm{KW}$ and three phase load terminal voltage is $363 \mathrm{~V}$.

b) Power system when DG to Bus-4:

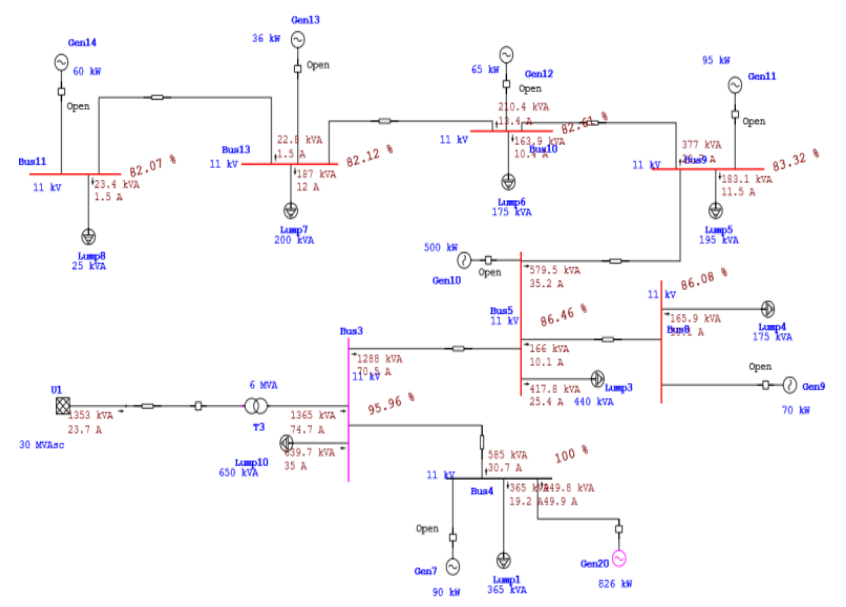

Figure 18: Load flow analysis of power system when DG to Bus 4

Table 9: Data of Load flow analysis of power system when DG to Bus 4.

\begin{tabular}{|c|c|c|c|c|c|c|c|c|}
\hline \multicolumn{9}{|c|}{ LOAD DATA } \\
\hline ID & Rating/Limit & Rated kV & kW & kvar & Amp & $\% \mathrm{PF}$ & $\begin{array}{c}\% \\
\text { Loading }\end{array}$ & Vterm \\
\hline Lump1 & $365 \mathrm{kVA}$ & 11 & 310.3 & 192.3 & 19.16 & 85 & 100 & 100 \\
\hline Lump3 & $440 \mathrm{kVA}$ & 11 & 355.1 & 220.1 & 25.36 & 85 & 109.8 & 86.46 \\
\hline Lump4 & $175 \mathrm{kVA}$ & 11 & 141 & 87.41 & 10.12 & 85 & 110.1 & 86.08 \\
\hline Lump5 & $195 \mathrm{kVA}$ & 11 & 155.6 & 96.44 & 11.53 & 85 & 112.7 & 83.32 \\
\hline Lump6 & $175 \mathrm{kVA}$ & 11 & 139.3 & 86.33 & 10.41 & 85 & 113.4 & 82.61 \\
\hline Lump7 & $200 \mathrm{kVA}$ & 11 & 158.9 & 98.49 & 11.95 & 85 & 113.8 & 82.12 \\
\hline Lump8 & $25 \mathrm{kVA}$ & 11 & 19.86 & 12.31 & 1.495 & 85 & 113.9 & 82.07 \\
\hline Lump10 & $650 \mathrm{kVA}$ & 11 & 543.8 & 337 & 34.99 & 85 & 102.6 & 95.96 \\
\hline & & & & & & & & 698.6 \\
\hline & & & & & & & & $87.32 i$ \\
\hline & & & & & & $\begin{array}{l}\text { 3 Phase } \\
\text { Load } \\
\text { Terminal } \\
\text { Voltage }\end{array}$ & & 349.2 \\
\hline \multicolumn{9}{|c|}{ SOURCE DATA } \\
\hline ID & $\begin{array}{c}\text { Terminal } \\
\text { Bus }\end{array}$ & Rating/Limit & Rated kV & MW & Mvar & Amp & $\% \mathrm{PF}$ & \\
\hline Gen20 & Bus4 & $0.826 \mathrm{MW}$ & 11 & 0.818 & 0.483 & 49.85 & 86.12 & \\
\hline U1 & Bus1 & $30 \mathrm{MVA}$ & 33 & 1.151 & 0.712 & 23.68 & 85.02 & \\
\hline & & & & & & & & \\
\hline & & & & & & & & \\
\hline
\end{tabular}

\begin{tabular}{|c|c|c|c|c|c|c|c|}
\hline \multicolumn{5}{|c|}{ BRANCH DATA } & & & \\
\hline ID & Type & kW Flow & kvar Flow & $\begin{array}{l}\text { Amp } \\
\text { Flow }\end{array}$ & & & \\
\hline Line1 & Line & 1121.3 & 806.7 & 24.89 & & & \\
\hline Line2 & Line & 492.4 & 275.5 & 30.86 & & & \\
\hline Line10 & Line & 986.6 & 616.3 & 70.62 & & & \\
\hline Line13 & Line & 141 & 87.41 & 10.12 & & & \\
\hline Line19 & Line & 476.5 & 294.2 & 35.28 & & & \\
\hline Line21 & Line & 318.9 & 195.9 & 23.78 & & & \\
\hline Line22 & Line & 178.8 & 109.6 & 13.41 & & & \\
\hline Line24 & Line & 19.86 & 12.31 & 1.494 & & & \\
\hline \multirow[t]{2}{*}{ T3 } & Transf. $2 \mathrm{~W}$ & 1121.3 & 806.7 & 24.89 & & & \\
\hline & & & & & \multicolumn{2}{|c|}{ GENERAL DATA } & \\
\hline \multicolumn{4}{|c|}{ BUS DATA } & & Buses & 10 & \\
\hline Bus ID & Nominal kV & Voltage & $\begin{array}{c}\text { MW } \\
\text { Loading }\end{array}$ & & Branches & 9 & \\
\hline Bus1 & 33 & 100 & 1.151 & & Generators & 1 & \\
\hline Bus3 & 11 & 95.96 & 1.611 & & Power Grids & 1 & \\
\hline Bus4 & 11 & 100 & 0.818 & & Loads & 8 & \\
\hline Bus5 & 11 & 86.46 & 0.987 & & & & \\
\hline Bus8 & 11 & 86.08 & 0.141 & & Load-MW & 1.969 & \\
\hline Bus9 & 11 & 83.32 & 0.477 & & Load-Mvar & 1.195 & \\
\hline Bus10 & 11 & 82.61 & 0.319 & & Generation-MW & 1.969 & \\
\hline Bus11 & 11 & 82.07 & 0.0199 & & $\begin{array}{l}\text { Generation- } \\
\text { Mvar }\end{array}$ & 1.195 & \\
\hline \multirow[t]{2}{*}{ Bus13 } & 11 & 82.12 & 0.179 & & Loss-MW & 0.145 & \\
\hline & & & & & Loss-Mvar & 0.0649 & \\
\hline
\end{tabular}


The Percentage voltage drop, load terminal voltage, KVA and current flowing at each bus is shown in Table 9 and Figure 18. From table, it is clear that he overall system losses after execution of load flow analysis when DG to Bus 4 was 145 $\mathrm{KW}$ and three phase load terminal voltage is $349 \mathrm{~V}$.

c) Power system when DG to Bus-5

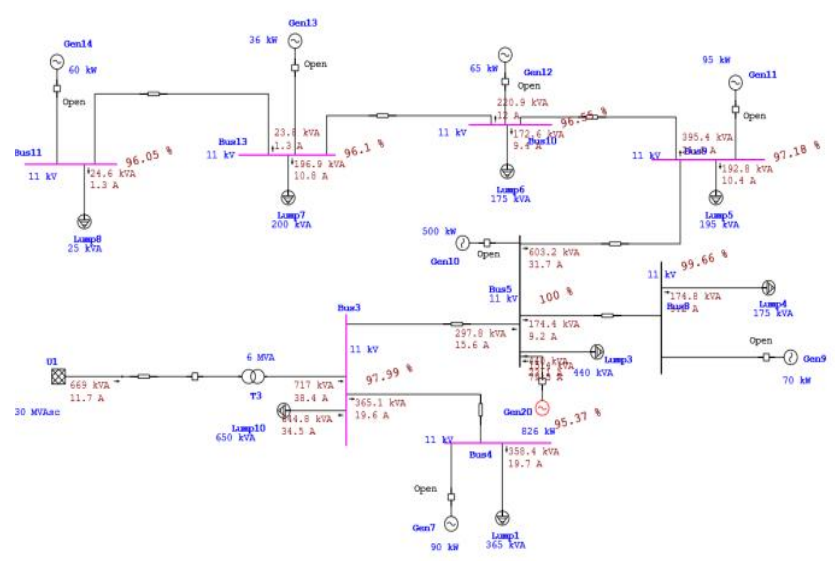

Figure 19: Load flow analysis of power system when DG to Bus 5

Table 10: Data of Load flow analysis of power system when DG to Bus 5.
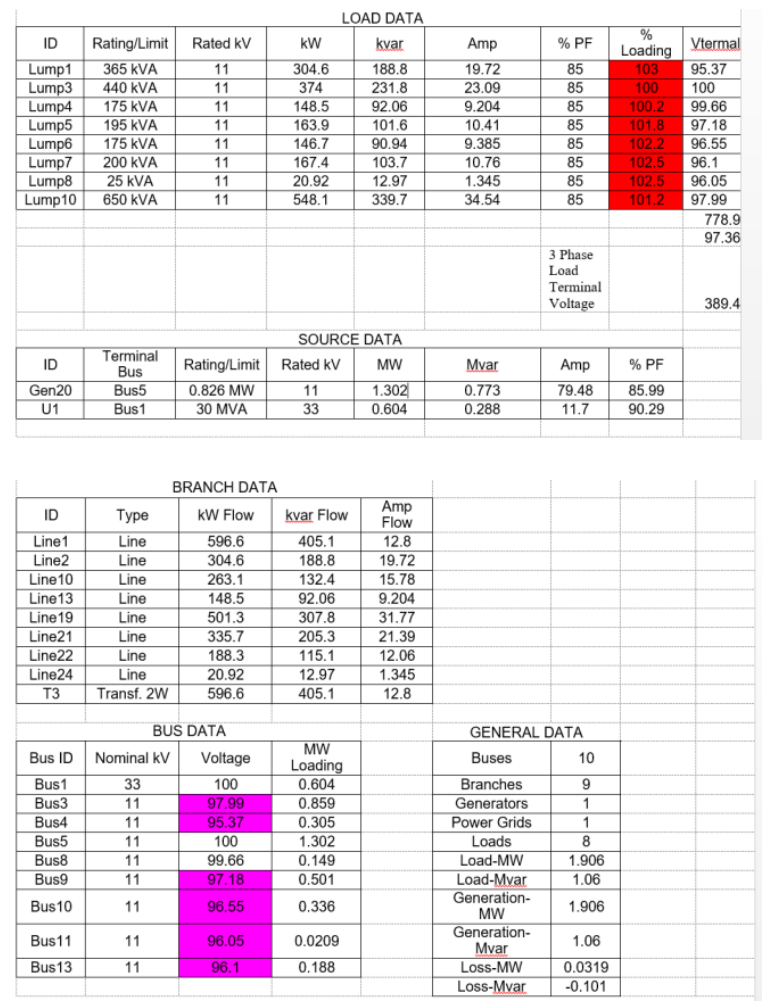

The Percentage voltage drop, load terminal voltage, KVA and current flowing at each bus is shown in Table 10 and Figure 19. From table, it is clear that he overall system losses after execution of load flow analysis when DG to Bus 5 was 31.9 $\mathrm{KW}$ and three phase load terminal voltage is $389 \mathrm{~V}$. d) Power system when DG to Bus-8

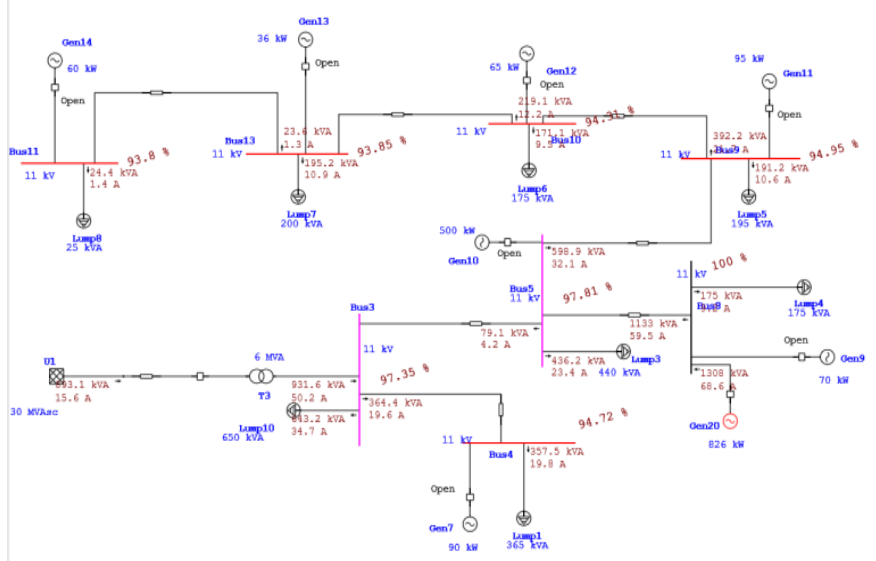

Figure 20: Load flow analysis of power system when DG to Bus 8

Table 11: Data of Load flow analysis of power system when DG to Bus 8 .

\begin{tabular}{|c|c|c|c|c|c|c|c|c|}
\hline \multicolumn{9}{|c|}{ LOAD DATA } \\
\hline ID & Rating/Limit & Rated kV & $\mathrm{kW}$ & kvar & Amp & $\% \mathrm{PF}$ & $\begin{array}{c}\% \\
\text { Loading }\end{array}$ & Vtermal \\
\hline Lump1 & $365 \mathrm{kVA}$ & 11 & 303.9 & 188.3 & 19.81 & 85 & 103.4 & 94.72 \\
\hline Lump3 & $440 \mathrm{kVA}$ & 11 & 370.8 & 229.8 & 23.41 & 85 & 101.4 & 97.81 \\
\hline Lump4 & $175 \mathrm{kVA}$ & 11 & 148.8 & 92.19 & 9.185 & 85 & 100 & 100 \\
\hline Lump5 & $195 \mathrm{kVA}$ & 11 & 162.5 & 100.7 & 10.57 & 85 & 103.2 & 94.95 \\
\hline Lump6 & $175 \mathrm{kVA}$ & 11 & 145.5 & 90.15 & 9.524 & 85 & 103.7 & 94.31 \\
\hline Lump7 & $200 \mathrm{kVA}$ & 11 & 165.9 & 102.8 & 10.92 & 85 & 104 & 93.85 \\
\hline Lump8 & $25 \mathrm{kVA}$ & 11 & 20.74 & 12.85 & 1.365 & 85 & 104 & 93.8 \\
\hline Lump10 & $650 \mathrm{kVA}$ & 11 & 546.7 & 338.8 & 34.68 & 85 & 101.6 & 97.35 \\
\hline & & & & & & & & 766.79 \\
\hline & & & & & & & & 95.849 \\
\hline & & & & & & \begin{tabular}{|l|}
3 Phase \\
Load \\
Terminal \\
Voltage
\end{tabular} & & 383.36 \\
\hline \multicolumn{9}{|c|}{ SOURCE DATA } \\
\hline ID & $\begin{array}{c}\text { Terminal } \\
\text { Bus }\end{array}$ & Rating/Limit & Rated kV & MW & Mvar & Amp & $\% \mathrm{PF}$ & \\
\hline Gen20 & Bus8 & $0.826 \mathrm{MW}$ & 11 & 1.122 & 0.673 & 68.65 & 85.75 & \\
\hline
\end{tabular}

\begin{tabular}{|c|c|c|c|c|c|c|c|c|}
\hline U1 & Bus1 & $30 \mathrm{MVA}$ & 33 & 0.794 & 0.41 & 15.63 & 88.85 & \\
\hline \multicolumn{5}{|c|}{ BRANCH DATA } & & & & \\
\hline ID & Type & kW Flow & kvar Flow & $\begin{array}{l}\text { Amp } \\
\text { Flow }\end{array}$ & & & & \\
\hline Line1 & Line & 780.6 & 521,4 & 16.74 & & & & \\
\hline Line2 & Line & 303.9 & 188.3 & 19.81 & & & & \\
\hline Line10 & Line & 77.62 & 19.42 & 4.314 & & & & \\
\hline Line13 & Line & 956.9 & 560.5 & 59.51 & & & & \\
\hline Line19 & Line & 497 & 305.5 & 32.25 & & & & \\
\hline Line21 & Line & 332.8 & 203.7 & 21.72 & & & & \\
\hline Line22 & Line & 186.7 & 114.2 & 12.24 & & & & \\
\hline Line24 & Line & 20.74 & 12.85 & 1.365 & & & & \\
\hline T3 & Transf. 2W & 780.6 & 521.4 & 16.74 & & & & \\
\hline & & & & & \multicolumn{2}{|c|}{ GENERAL DATA } & & \\
\hline \multicolumn{4}{|c|}{ BUS DATA } & & Buses & 10 & & \\
\hline Bus ID & Nominal $\mathrm{kV}$ & Voltage & $\begin{array}{c}\text { MW } \\
\text { Loading }\end{array}$ & & Branches & 9 & & \\
\hline Bus1 & 33 & 100 & 0.794 & & Generators & 1 & & \\
\hline Bus3 & 11 & 97.35 & 0.857 & & Power Grids & 1 & & \\
\hline Bus4 & 11 & 94.72 & 0.304 & & Loads & 8 & & \\
\hline Bus5 & 11 & 97.81 & 0.957 & & & & & \\
\hline Bus8 & 11 & 100 & 1.122 & & Load-MW & 1.915 & & \\
\hline Bus9 & 11 & 94.95 & 0.497 & & Load-Mvar & 1.083 & & \\
\hline Bus10 & 11 & 94.31 & 0.333 & & $\begin{array}{l}\text { Generation- } \\
\text { MW }\end{array}$ & 1.915 & & \\
\hline Bus11 & 11 & 93.8 & 0.0207 & & $\begin{array}{l}\text { Generation- } \\
\text { Mvar }\end{array}$ & 1.083 & & \\
\hline \multirow[t]{2}{*}{ Bus13 } & 11 & 93.85 & 0.187 & & Loss-MW & 0.0503 & & \\
\hline & & & & & Loss-Mvar. & -0.073 & & \\
\hline
\end{tabular}

The Percentage voltage drop, load terminal voltage, KVA and current flowing at each bus is shown in Table 11 and Figure 20. From table, it is clear that he overall system losses after execution of load flow analysis when DG to Bus 8 was 50.3 $\mathrm{KW}$ and three phase load terminal voltage is $383 \mathrm{~V}$. 
e) Power system when DG to Bus-9

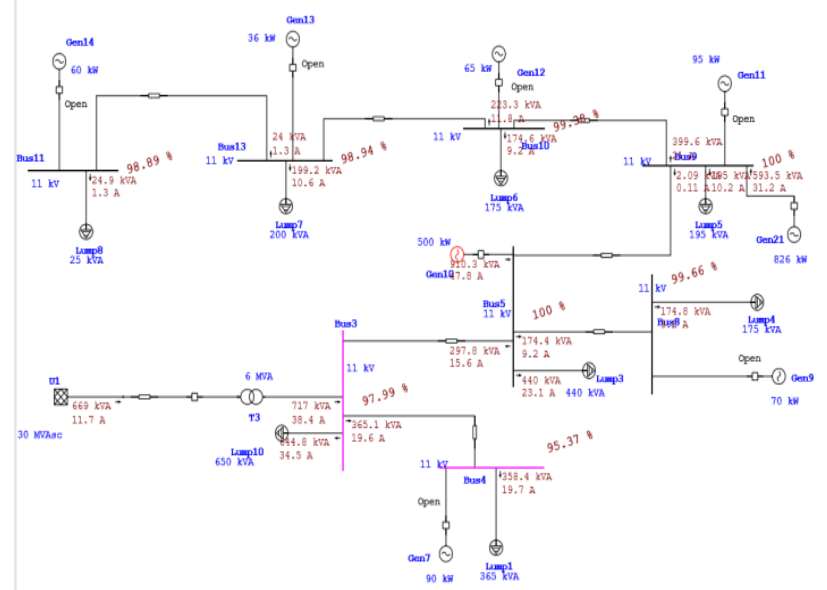

Figure 21: Load flow analysis of power system when DG to Bus 9

Table 12: Data of Load flow analysis of power system when DG to Bus 9.

\begin{tabular}{|c|c|c|c|c|c|c|c|c|}
\hline \multicolumn{9}{|c|}{ LOAD DATA } \\
\hline ID & Rating/Limit & Rated kV & $\mathrm{kW}$ & kvar & Amp & $\% \mathrm{PF}$ & $\begin{array}{c}\% \\
\text { Loading }\end{array}$ & Vtermal \\
\hline Lump1 & $365 \mathrm{kVA}$ & 11 & 303.6 & 188.2 & 19.84 & 85 & 103.5 & 94.51 \\
\hline Lump3 & $440 \mathrm{kVA}$ & 11 & 369.7 & 229.1 & 23.51 & 85 & 101.8 & 97.11 \\
\hline Lump4 & $175 \mathrm{kVA}$ & 11 & 146.9 & 91.01 & 9.371 & 85 & 102 & 96.77 \\
\hline Lump5 & $195 \mathrm{kVA}$ & 11 & 165.8 & 102.7 & 10.23 & 85 & 100 & 100 \\
\hline Lump6 & $175 \mathrm{kVA}$ & 11 & 148.4 & 91.96 & 9.22 & 85 & 100.4 & 99.38 \\
\hline Lump7 & $200 \mathrm{kVA}$ & 11 & 169.3 & 104.9 & 10.56 & 85 & 100.6 & 98.94 \\
\hline Lump8 & $25 \mathrm{kVA}$ & 11 & 21.16 & 13.11 & 1.321 & 85 & 100.7 & 98.89 \\
\hline Lump10 & $650 \mathrm{kVA}$ & 11 & 546.3 & 338.6 & 34.72 & 85 & 101.8 & 97.15 \\
\hline & & & & & & & & 782.75 \\
\hline & & & & & & & & 97.8 \\
\hline & & & & & & & $\begin{array}{l}3 \text { Phase } \\
\text { Load } \\
\text { Terminal } \\
\text { Voltage }\end{array}$ & 391.2 \\
\hline \multicolumn{9}{|c|}{ SOURCE DATA } \\
\hline ID & $\begin{array}{c}\text { Terminal } \\
\text { Bus }\end{array}$ & Rating/Limit & Rated kV & MW & Mvar & Amp & $\%$ PF & \\
\hline Gen20 & Bus9 & $0.826 \mathrm{MW}$ & 11 & 1.05 & 0.623 & 64.08 & 86.02 & \\
\hline U1 & Bus1 & 30 MVA & 33 & 0.858 & 0.446 & 16.92 & 88.71 & \\
\hline
\end{tabular}

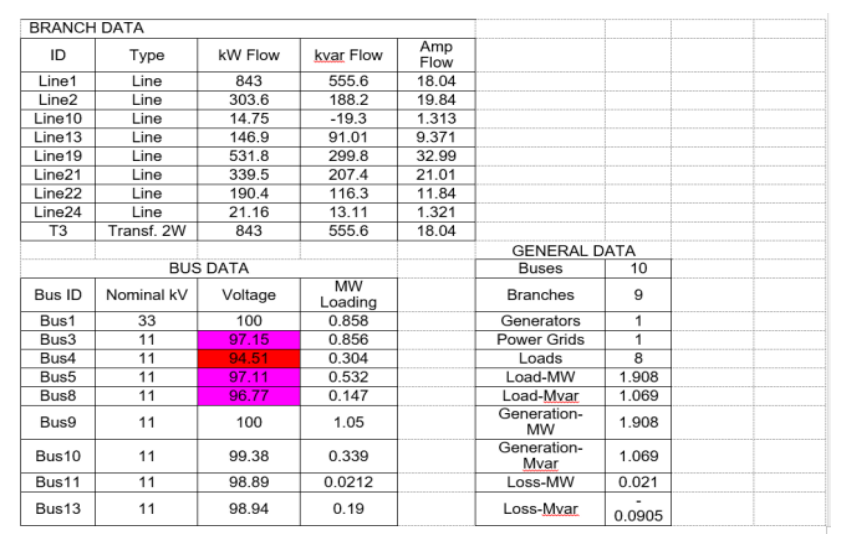

The Percentage voltage drop, load terminal voltage, KVA and current flowing at each bus is shown in Table 12 and Figure 21. From table, it is clear that he overall system losses after execution of load flow analysis when DG to Bus 9 was $21 \mathrm{KW}$ and three phase load terminal voltage is $391.2 \mathrm{~V}$.

\section{f) Power system with DG to Bus-10}

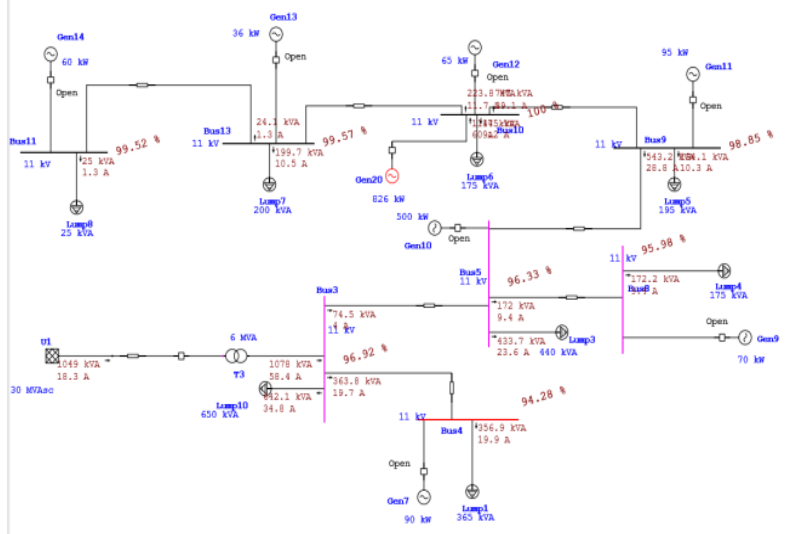

Figure 22: Load flow analysis of power system when DG to Bus 10

Table 13: Data of Load flow analysis of power system when DG to Bus 10

\begin{tabular}{|c|c|c|c|c|c|c|c|c|}
\hline \multicolumn{9}{|c|}{ LOAD DATA } \\
\hline ID & Rating/Limit & Rated kV & kW & kvar & Amp & $\% \mathrm{PF}$ & $\begin{array}{c}\% \\
\text { Loading }\end{array}$ & Vterma \\
\hline Lump1 & $365 \mathrm{kVA}$ & 11 & 303.4 & 188 & 19.87 & 85 & 103.7 & 94.28 \\
\hline Lump3 & $440 \mathrm{kVA}$ & 11 & 368.6 & 228.4 & 23.63 & 85 & 102.3 & 96.33 \\
\hline Lump4 & $175 \mathrm{kVA}$ & 11 & 146.4 & 90.73 & 9.419 & 85 & 102.5 & 95.98 \\
\hline Lump5 & $195 \mathrm{kVA}$ & 11 & 165 & 102.3 & 10.31 & 85 & 100.7 & 98.85 \\
\hline Lump6 & $175 \mathrm{kVA}$ & 11 & 148.8 & 92.19 & 9.185 & 85 & 100 & 100 \\
\hline Lump7 & $200 \mathrm{kVA}$ & 11 & 169.7 & 105.2 & 10.52 & 85 & 100.3 & 99.57 \\
\hline Lump8 & $25 \mathrm{kVA}$ & 11 & 21.21 & 13.14 & 1.316 & 85 & 100.3 & 99.52 \\
\hline Lump10 & $650 \mathrm{kVA}$ & 11 & 545.8 & 338.3 & 34.77 & 85 & 101.9 & 96.92 \\
\hline & & & & & & & & 781.45 \\
\hline & & & & & & & & 97.68 \\
\hline & & & & & & $\begin{array}{l}3 \text { Phase } \\
\text { Load } \\
\text { Terminal } \\
\text { Voltage }\end{array}$ & & 390 \\
\hline \multicolumn{9}{|c|}{ SOURCE DATA } \\
\hline ID & $\begin{array}{c}\text { Terminal } \\
\text { Bus }\end{array}$ & Rating/Limit & $\begin{array}{l}\text { Rated } \\
\mathrm{kV}\end{array}$ & MW & Mvar. & Amp & $\%$ PF & \\
\hline Gen20 & Bus10 & $0.826 \mathrm{MW}$ & 11 & 0.983 & 0.584 & 60.03 & 85.97 & \\
\hline U1 & Bus1 & 30 MVA & 33 & 0.927 & 0.491 & 18.35 & 88.39 & \\
\hline
\end{tabular}

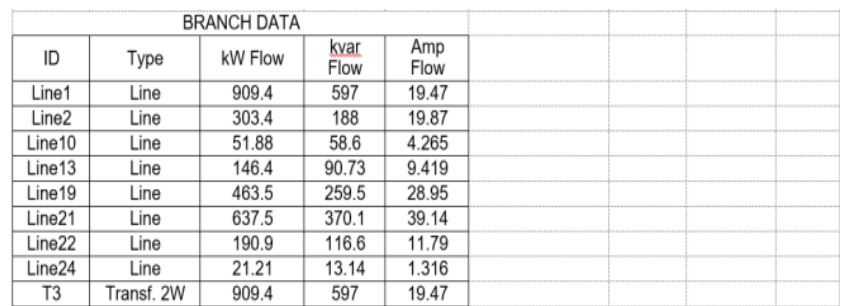

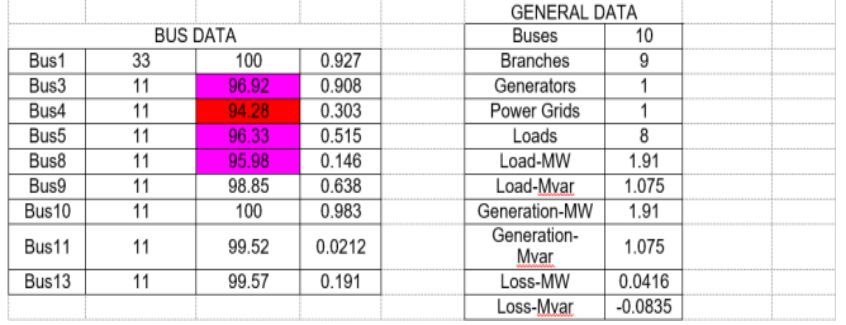

The Percentage voltage drop, load terminal voltage, KVA and current flowing at each bus is shown in Table 13 and Figure 22. From table, it is clear that he overall system losses after execution of load flow analysis when DG to Bus 10 was 41.6 KW and three phase load terminal voltage is $390 \mathrm{~V}$. 


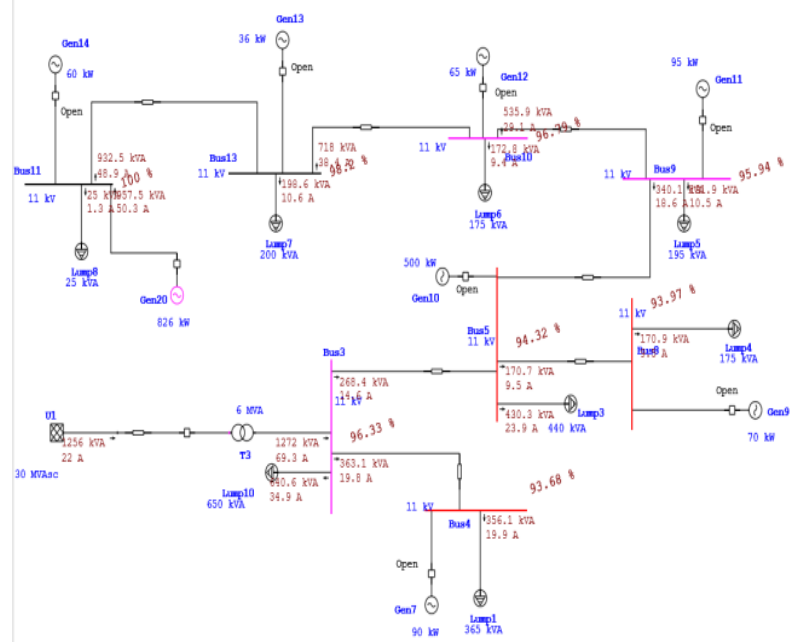

Figure 23: Load flow analysis of power system when DG to Bus 11

Table 14: Data of Load flow analysis of power system when DG to Bus 11.

\begin{tabular}{|c|c|c|c|c|c|c|c|c|}
\hline \multicolumn{9}{|c|}{ LOAD DATA } \\
\hline ID & Rating/Limit & Rated kV & $\mathrm{kW}$ & kvar & Amp & $\% \mathrm{PF}$ & $\begin{array}{c}\% \\
\text { Loading }\end{array}$ & Vtermal \\
\hline Lump1 & 365 kVA & 11 & 302.7 & 187.6 & 19.95 & 85 & 104.1 & 93.68 \\
\hline Lump3 & $440 \mathrm{kVA}$ & 11 & 365.7 & 226.7 & 23.94 & 85 & 103.7 & 94.32 \\
\hline Lump4 & $175 \mathrm{kVA}$ & 11 & 145.3 & 90.03 & 9.546 & 85 & 103.9 & 93.97 \\
\hline Lump5 & $195 \mathrm{kVA}$ & 11 & 163.1 & 101.1 & 10.5 & 85 & 102.6 & 95.94 \\
\hline Lump6 & 175 kVA & 11 & 146.9 & 91.02 & 9.37 & 85 & 102 & 96.79 \\
\hline Lump7 & 200 kVA & 11 & 168.8 & 104.6 & 10.61 & 85 & 101.1 & 98.2 \\
\hline Lump8 & $25 \mathrm{kVA}$ & 11 & 21.25 & 13.17 & 1.312 & 85 & 100 & 100 \\
\hline Lump10 & $650 \mathrm{kVA}$ & 11 & 544.5 & 337.5 & 34.91 & 85 & 102.3 & 96.33 \\
\hline & & & & & & & & 769.23 \\
\hline & & & & & & & & 96.1538 \\
\hline & & & & & & $\begin{array}{l}3 \text { Phase } \\
\text { Load } \\
\text { Terminal } \\
\text { Voltage }\end{array}$ & & 384.6 \\
\hline \multicolumn{9}{|c|}{ SOURCE DATA } \\
\hline ID & $\begin{array}{c}\text { Terminal } \\
\text { Bus }\end{array}$ & Rating/Limit & Rated kV & MW & Mvar & Amp & $\% \mathrm{PF}$ & \\
\hline Gen20 & Bus11 & $0.826 \mathrm{MW}$ & 11 & 0.82 & 0.495 & 50.26 & 85.62 & \\
\hline
\end{tabular}

\begin{tabular}{|c|c|c|c|c|c|c|c|}
\hline U1 & Bus1 & 30 MVA & 33 & 1.101 & 0.605 & 21.97 & 87.64 \\
\hline \multicolumn{5}{|c|}{ BRANCH DATA } & & & \\
\hline ID & Type & kW Flow & kvar Flow & $\begin{array}{l}\text { Amp } \\
\text { Flow }\end{array}$ & & & \\
\hline Line1 & Line & 1075.4 & 703.4 & 23.1 & & & \\
\hline Line2 & Line & 302.7 & 187.6 & 19.95 & & & \\
\hline Line10 & Line & 215.8 & 155.6 & 14.81 & & & \\
\hline Line13 & Line & 145.3 & 90.03 & 9.546 & & & \\
\hline Line19 & Line & 295.6 & 160.2 & 18.71 & & & \\
\hline Line21 & Line & 462.4 & 262.6 & 29.09 & & & \\
\hline Line22 & Line & 612.4 & 356.5 & 38.42 & & & \\
\hline Line24 & Line & 787.8 & 468.5 & 48.99 & & & \\
\hline \multirow[t]{2}{*}{ T3 } & Transf. 2W & 1075.4 & 703.4 & 23.1 & & & \\
\hline & & & & & \multicolumn{2}{|c|}{ GENERAL DATA } & \\
\hline \multicolumn{4}{|c|}{ BUS DATA } & & Buses & 10 & \\
\hline Bus ID & Nominal kV & Voltage & $\begin{array}{l}\text { MW } \\
\text { Loading }\end{array}$ & & Branches & 9 & \\
\hline Bus1 & 33 & 100 & 1.101 & & Generators & 1 & \\
\hline Bus3 & 11 & 96.33 & 1.073 & & Power Grids & 1 & \\
\hline Bus4 & 11 & 93.68 & 0.303 & & Loads & 8 & \\
\hline Bus5 & 11 & 94.32 & 0.511 & & Load-MW & 1.92 & \\
\hline Bus8 & 11 & 93.97 & 0.145 & & Load-Mvar & 1.1 & \\
\hline Bus9 & 11 & 95.94 & 0.462 & & $\begin{array}{l}\text { Generation- } \\
\text { MW }\end{array}$ & 1.92 & \\
\hline Bus10 & 11 & 96.79 & 0.612 & & $\begin{array}{l}\text { Generation- } \\
\text { Mvar }\end{array}$ & 1.1 & \\
\hline Bus11 & 11 & 100 & 0.82 & & Loss-MW & 0.0621 & \\
\hline Bus13 & 11 & 98.2 & 0.788 & & Loss-Mvar & -0.0521 & \\
\hline
\end{tabular}

The Percentage voltage drop, load terminal voltage, KVA and current flowing at each bus is shown in Table 14 and Figure 23. From table, it is clear that he overall system losses after execution of load flow analysis when DG to Bus 11 was 62 $\mathrm{KW}$ and three phase load terminal voltage is $384.6 \mathrm{~V}$.

h)

Power system when DG to Bus-13

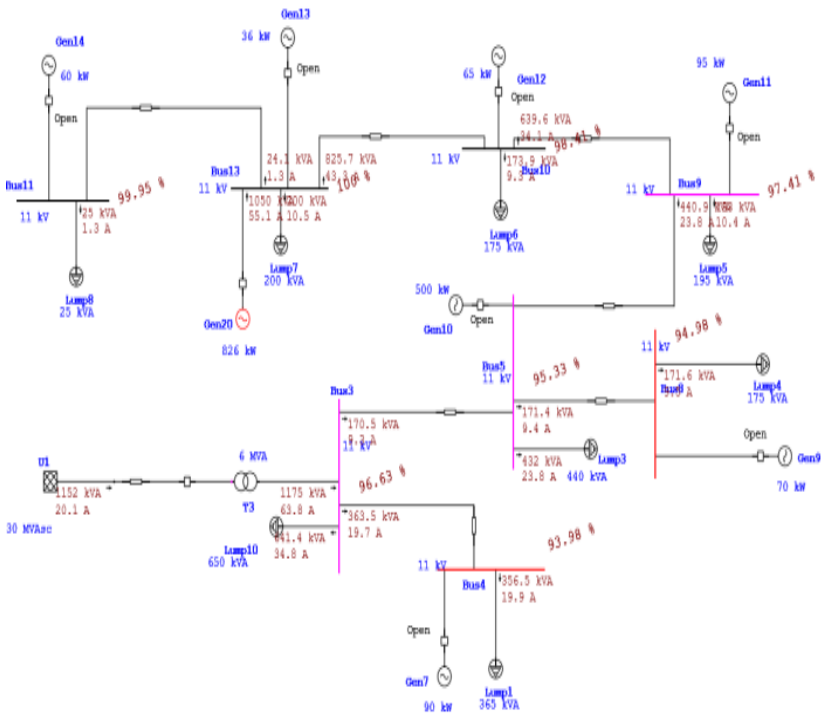

Figure 24: Load flow analysis of power system when DG to Bus 13

Table 15: Data of Load flow analysis of power system when DG to Bus 13.

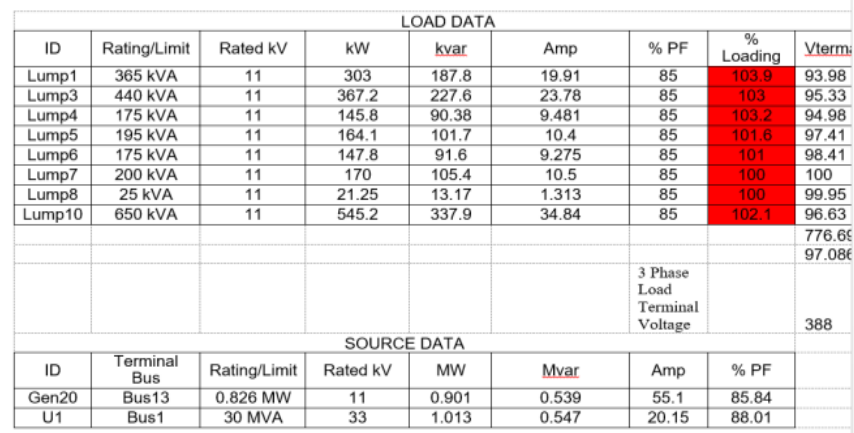

\begin{tabular}{|c|c|c|c|c|c|c|c|c|}
\hline \multicolumn{5}{|c|}{ BRANCH DATA } & & & & \\
\hline ID & Type & kW Flow & kvar Flow & $\begin{array}{l}\text { Amp } \\
\text { Flow }\end{array}$ & & & & \\
\hline Line1 & Line & 992.2 & 649.5 & 21.27 & & & & \\
\hline Line2 & Line & 303 & 187.8 & 19.91 & & & & \\
\hline Line10 & Line & 134.1 & 107.2 & 9.454 & & & & \\
\hline Line13 & Line & 145.8 & 90.38 & 9.481 & & & & \\
\hline Line19 & Line & 379.3 & 209.7 & 23.86 & & & & \\
\hline Line21 & Line & 549.5 & 315.9 & 34.15 & & & & \\
\hline Line22 & Line & 701.5 & 411.9 & 43.39 & & & & \\
\hline Line24 & Line & 21.25 & 13.17 & 1.313 & & & & \\
\hline T3 & Transf. 2W & 992.2 & 649.5 & 21.27 & & & & \\
\hline \multirow{2}{*}{\multicolumn{4}{|c|}{ BUS DATA }} & & \multicolumn{2}{|c|}{ GENERAL DATA } & & \\
\hline & & & & & Buses & 10 & & \\
\hline Bus ID & Nominal kV & Voltage & $\begin{array}{l}\text { MW } \\
\text { Loading }\end{array}$ & & Branches & 9 & & \\
\hline Bus1 & 33 & 100 & 1.013 & & Generators & 1 & & \\
\hline Bus3 & 11 & 96.63 & 0.99 & & Power Grids & 1 & & \\
\hline Bus4 & 11 & 93.98 & 0.303 & & Loads & 8 & & \\
\hline Bus5 & 11 & 95.33 & 0.513 & & Load-MW & 1.915 & & \\
\hline Bus8 & 11 & 94.98 & 0.146 & & Load-Mvar & 1.085 & & \\
\hline Bus9 & 11 & 97.41 & 0.549 & & Generation-MW & 1.915 & & \\
\hline Bus10 & 11 & 98.41 & 0.701 & & $\begin{array}{l}\text { Generation- } \\
\text { Mvar }\end{array}$ & 1.085 & & \\
\hline Bus11 & 11 & 99.95 & 0.0212 & & Loss-MW & 0.0503 & & \\
\hline Bus13 & 11 & 100 & 0.901 & & Loss-Mvar & -0.0701 & & \\
\hline
\end{tabular}


The Percentage voltage drop, load terminal voltage, KVA and current flowing at each bus is shown in Table 15 and Figure 24. From table, it is clear that he overall system losses after execution of load flow analysis when DG to Bus 13 was 50.3 $\mathrm{KW}$ and three phase load terminal voltage is $388 \mathrm{~V}$.

\section{COMPARISON}

Comparing Power Losses and Voltages at Different Busses after Installation of DG, the summary is presented in the table 16 below.

Table 16: Comparison Of Losses and voltage Data of Load flow analysis of power system when DG installation to Different Bus.

\begin{tabular}{|l|l|l|l|}
\hline \multicolumn{1}{|c|}{ Bus ID } & Nominal kV in KV & $\begin{array}{c}\text { Load terminal } \\
\text { Voltage in V }\end{array}$ & \multicolumn{1}{c|}{ Losses in KW } \\
\hline Bus2 & 33 & & 0 \\
\hline Bus3 & 11 & 363 & 96.7 \\
\hline Bus4 & 11 & 349 & 145 \\
\hline Bus5 & 11 & 389 & 31.9 \\
\hline Bus 8 & 11 & 383 & 50.3 \\
\hline Bus 9 & 11 & 391 & 21 \\
\hline Bus 10 & 11 & 390 & 41.6 \\
\hline Bus 11 & 11 & 384 & 62.1 \\
\hline Bus 13 & 11 & 388 & 50.3 \\
\hline
\end{tabular}

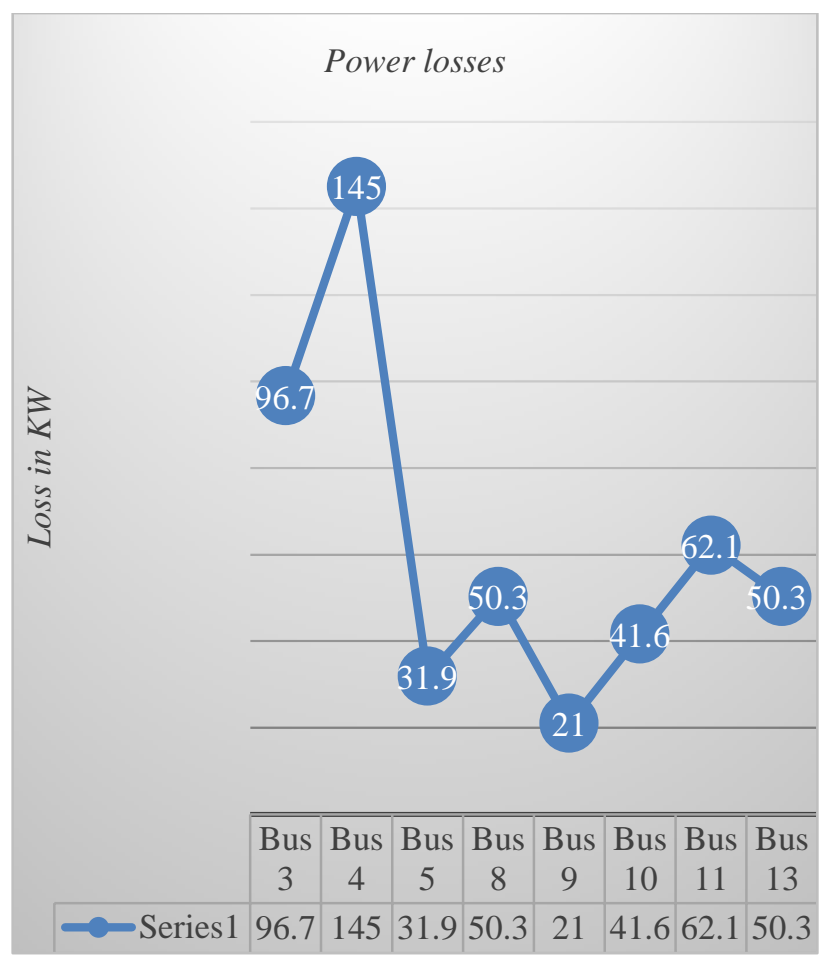

Figure 25: Power Losses at Different Busses after Installation of DG

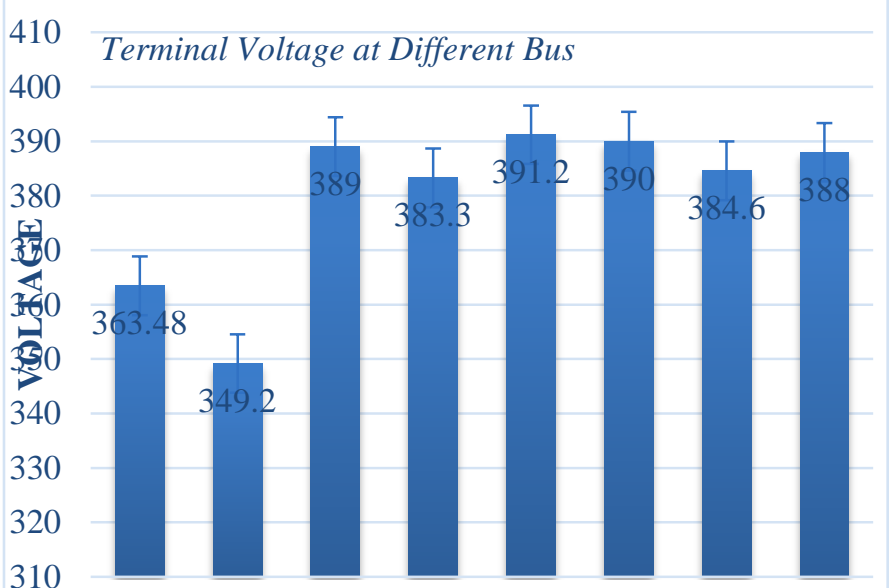

Bus 3 Bus 4 Bus 5 Bus 8 Bus 9 Bus 10Bus 11Bus 13

- Series1

Figure 26: Voltage at Different Busses after Installation of DG

Above results of simulation show the losses of the power system. DG is connected at different buses step by step and Bus-9 is the only bus where losses of the power system are least recorded which is $21 \mathrm{KW}$ and voltage of bus 9 is highest among the buses which is $391.2 \mathrm{~V}$, therefore, the Bus-9 bus is the optimal placement of distributed generation for power system. Consequently, the Bus-9 is the optimal placement of the system because at this location the voltage profile is generally improved while losses are drastically minimized.

\section{CONCLUSION}

Demonstration of the grid impact study shows the losses on lines as well as percentage voltage drop on the network decreases with the increase in power export from Mini grid and MHP Plant. Terminal voltage at different load point also get improved with increase in power export from Mini grid and MHP Plant. The overarching view from the results is that the outlook for Local Distribution grid interconnected mini grid and MHP plant in future is positive in Nepal.

In this research, detailed analysis on some of the impacts of distributed generation (DG) on a distribution network operation is conducted.

The obtained results have shown that the DG influences the distribution network and that their precise location are vital in reducing power losses and improving the voltage Profile. It is noted that DG placement cannot always results to effective loss reduction i.e., it depends on the location of DG unit. DG implementation as a source of active power has a great positive impact on improving the voltage profile through the entire distribution network.

\section{ACKNOWLEDGEMENT}

The authors extend their hearty gratitude to the faculties and colleagues at Tribhuvan University, Institute of Engineering, 
Pulchowk Campus and engineers of Nepal Electricity Authority for their constant help, support and recommendations.

\section{REFERENCES}

[1] Aepc, 2018/19. Progress at a Glance :A year in Review. s.1., s.n.

[2] District development commiunity, t., 2016. Taplejung mini grid development project, s.1.: s.n.

[3] Gyawali, n., 2014. Analysis and design of Taplejung mini grid, s.1.: Multi Electrical Solutions.

[4] NEA, 2018/19. A year in review -fiscal year, s.l.: s.n.

[5] Ray, S. \& Jha, A. K., 2017. Grid Integration Studies of Captive and Cogeneration Plant in Sugar Industry: Case Study of Indushankar Sugar Industry Pvt. Ltd.. Kathmandu, s.n.

[6] Paudel, B. \& Gyawali, N., 2016. Improvement of Power Control Strategy for Islanded Microgrid Power System. Kathmandu, s.n.

[7] Thapa, K. et al., 2018. Modelling of wind-solar Hybrid Power System for Off-Grid in Nepal and a case study. Kathmandu, RETRUD-18.

[8] Tiwari, B. R., Bhattarai, N. \& Jha, A. K., 2017. Performance analysis of a $100 \mathrm{kWp}$ grid connected Solar Photovoltaic Power Plant in Kharipati, Bhaktapur, Nepal. s.l., s.n.

[9] World Bank Group, n.d. Nepal: Scaling Up Electricity Access through Mini and Micro Hydropower Applications, s.l.: s.n.

[10] Shukla, T., 2010. Allocation of optimal distributed generation using ga for minimum system inradial distribution networks. s.l., s.n.

[11] Shrestha, J. N. \& Raut, D. B., 2018. Assessment of Urban roof top Grid Connected Solar Potential in Nepal. Kathmandu, Retrud_18.

[12] Okhueleigbe, O. P. a. E. I., 2017. Optimal Placement of Distributed Generation (DG) in a Distribution Network. s.1., International Journal of Latest Research in Engineering and Technology (IJLRET) .

[13] mallik, j. k., 2019. Micro Hydro Power in Nepal: A Journey from stand alone system to distributed generation. s.l., s.n.

[14] Kothari, D. P., 2017. Modern power system. Fourth Edition ed. s.1.:Mc graw-hill.

[15] Kundor, P., Mc graw-hill,1994. Powersystem stability and control. s.l., s.n.

[16] Bhandari, K. R. \& Adhikari, N. P., 2018. Grid integration of Solar and Solar/Wind hybrid Mini-Grid Projects: A case of Solar/Wind Hybrid MiniGrid Project Implemented by AEPC. Kathmandu, Retrud-18.
[17] Aepc/Rerl, December,2015. Detail study of transmission survey/mapping/design and cost estimation of seven mhp for mini grid formation in Taplejung district., Smart consult pvt.ltd.volume -1.

[18] Pabla,A.S., Mc graw-hill,Electric power planning, second Edition

Rajmani Bajagain, Nawraj Bhattarai, Tek Raj Subedi: Department of Mechanical Engineering, Institute of Engineering, Pulchowk Campus, Tribhuvan University, Nepal 